\title{
Difference operators for partitions under the Littlewood decomposition
}

\author{
Dehaye, Paul-Olivier ; Han, Guo-Niu ; Xiong, Huan
}

\begin{abstract}
The concept of $t$-difference operator for functions of partitions is introduced to prove a generalization of Stanley's theorem on polynomiality of Plancherel averages of symmetric functions related to contents and hook lengths. Our extension uses a generalization of the notion of Plancherel measure, based on walks in the Young lattice with steps given by the addition of $t$-hooks. It is well known that the hook lengths of multiples of $t$ can be characterized by the Littlewood decomposition. Our study gives some further information on the contents and hook lengths of other congruence classes modulo $t$.
\end{abstract}

DOI: https://doi.org/10.1007/s11139-016-9807-z

Posted at the Zurich Open Repository and Archive, University of Zurich

ZORA URL: https://doi.org/10.5167/uzh-131046

Journal Article

Accepted Version

Originally published at:

Dehaye, Paul-Olivier; Han, Guo-Niu; Xiong, Huan (2017). Difference operators for partitions under the Littlewood decomposition. The Ramanujan Journal, 44(1):197-225.

DOI: https://doi.org/10.1007/s11139-016-9807-z 


\title{
DIFFERENCE OPERATORS FOR PARTITIONS UNDER THE LITTLEWOOD DECOMPOSITION
}

\author{
PAUL-OLIVIER DEHAYE, GUO-NIU HAN AND HUAN XIONG
}

\begin{abstract}
The concept of $t$-difference operator for functions of partitions is introduced to prove a generalization of Stanley's theorem on polynomiality of Plancherel averages of symmetric functions related to contents and hook lengths. Our extension uses a generalization of the notion of Plancherel measure, based on walks in the Young lattice with steps given by the addition of $t$-hooks. It is well-known that the hook lengths of multiples of $t$ can be characterized by the Littlewood decomposition. Our study gives some further information on the contents and hook lengths of other congruence classes modulo $t$.
\end{abstract}

\section{INTRODUCTION}

1.1. Goal. The purpose of this paper is to compute explicit averages over partitions of some statistics of their invariants, such as contents and hook lengths, and establish polynomiality of these averages in the size of the partitions considered. In contrast with previous results, we restrict our attention to subsets of partitions classified by the divisibility properties of those invariants, and we modify the averaging measures accordingly. Our main result is stated in Theorem 1.3.

1.2. Basic definitions. A partition is a finite weakly decreasing sequence of positive integers $\lambda=\left(\lambda_{1}, \lambda_{2}, \cdots, \lambda_{r}\right)$. The size of the partition $\lambda$ is defined by the integer $|\lambda|=\sum_{1 \leq i \leq r} \lambda_{i}$. A partition $\lambda$ could be represented by its Young diagram, which is a collection of boxes arranged in left-justified rows with $\lambda_{i}$ boxes in the $i$-th row. The content of a box $\square=(i, j)$ in a partition $\lambda$ is defined by $c_{\square}=j-i$. With each $(i, j)$-box is associated its hook length, denoted by $h_{(i, j)}$, which is the number of boxes exactly to the right, or exactly below, including the box itself. The hook length multi-set of $\lambda$ is denoted by $\mathcal{H}(\lambda)$. In Figure 1 is represented the Young diagram and hook lengths of the partition $(6,3,2,2)$. For example, $h_{(1,2)}=8$ and $h_{(3,1)}=3$.

\begin{tabular}{|c|c|c|c|c|}
\hline 9 & 8 & 5 & \begin{tabular}{l|l}
3 & 2
\end{tabular} & . \\
\hline 5 & 4 & 1 & & \\
\hline 3 & 2 & & & \\
\hline 2 & 1 & & & \\
\hline
\end{tabular}

Figure 1. The Young diagram of the partition $(6,3,2,2)$ and the hook lengths of corresponding boxes.

2010 Mathematics Subject Classification. 05A15, 05A17, 05A19, 05E05, 05E10, 11P81.

Key words and phrases. Littlewood decomposition, partition, Plancherel measure, Difference operator. 
Let $t$ be a positive integer. A partition is called a $t$-core partition if none of its hook lengths is divisible by $t$. For example, from Figure 1 we can see that $\lambda=(6,3,2,2)$ is a 7 -core partition. Let

$$
\mathcal{H}_{t}(\lambda)=\{h \in \mathcal{H}(\lambda): h \equiv 0 \quad(\bmod t)\}
$$

be the multi-set of hook lengths of multiples of $t$.

\subsection{1-sequences, Littlewood decomposition and Plancherel averages.} Each partition $\lambda$ can be associated with its 01-sequence $z(\lambda)=\left(z_{\lambda, i}\right)_{i \in \mathbb{Z}}=\left(z_{i}\right)_{i \in \mathbb{Z}}$ as follows. First, consider the edges in the boundary of $\lambda$, starting at the bottom and ending to the right. Label the vertical (resp. horizontal) edges 0 (resp. 1). We obtain a bi-infinite 01-sequence $\left(z_{i}\right)_{i \in \mathbb{Z}}$ beginning with infinitely many 0's and ending with infinitely many 1 's. The index 0 of the bi-infinite 01 -sequence is defined by the condition $\#\left\{z_{i}=0: i \geq 0\right\}=\#\left\{z_{i}=1: i<0\right\}$. Every partition and its 01-sequence are uniquely determined by each other (see Figure 2 for a running example of the constructions presented in this section).

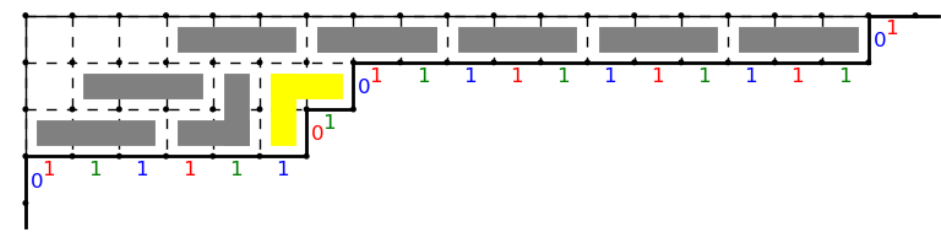

Figure 2. This is our running example for the 01-sequence and the Littlewood decomposition of a partition. The Young diagram of the partition $\lambda=(18,7,6)$ is drawn, together with its 01-sequence ‥0111|1110101111111111101… (the $\mid$ indicates the unique location where the number of 1's to the left equals the number of 0 's to the right, which is between index -1 and 0 in the 01-sequence; visually this corresponds to the main diagonal in the Young diagram). Notice the mnemonic "RGB" for the colourings, starting after the $\mid$ mark. The grey shapes indicate a sequence of 3 -hooks that can be removed from $\lambda$, to finally obtain the 3-core $(3,1)$ of $\lambda$. One could start with the yellow shape for instance, and then make various choices for the order of the removals. The process always ends with the same 3 -core $(3,1)$, irrespective of the order of removals. Quotients will be $\cdots 01 \mid 1011111 \cdots$ (partition $(2)), \cdots 0 \mid 1111111 \cdots($ empty partition $\emptyset)$ and $\cdots 011 \mid 011101 \cdots$ (partition $(5,2))$.

The 01-sequences contain a lot of information about partitions (see $[2,12])$.

Lemma 1.1. For a partition $\lambda$ and its 01-sequence $z(\lambda)=\left(z_{i}\right)_{i \in \mathbb{Z}}$, we have

$$
|\lambda|=\#\left\{(i, j): i<j, z_{i}=1, z_{j}=0\right\} .
$$

Actually, each box in $\lambda$ is uniquely determined by such a pair $(i, j) .{ }^{1}$ The hook length of such box is $j-i$. Also, for an edge labeled by $z_{i}=0$, the box to the left has content $i$; for an edge labeled by $z_{i}=1$, the box above has content $i+1$.

${ }^{1}$ Notice that here $(i, j)$ is not the coordinate of the box in $\lambda$. 
By Lemma 1.1 a partition $\lambda$ is a $t$-core partition if and only if there are no $i \in \mathbb{Z}$ satisfying $z_{i}=1, z_{i+t}=0$ in the 01-sequence $z(\lambda)$. If $z_{i}=1, z_{i+t}=0$ (resp. $z_{i}=0, z_{i+t}=1$ ) in a 01-sequence $z(\lambda)=\left(z_{j}\right)_{j \in \mathbb{Z}}$, by exchanging the values of $z_{i}$ and $z_{i+t}$ we obtain a new 01-sequence and thus a new partition. We say that this new partition is obtained by removing (resp. adding) a $t$-hook from (resp. to) $\lambda$.

Starting with a partition $\lambda$, we can always remove several $t$-hooks until no more $t$-hooks could be removed. The final partition we get by this operation is called the $t$-core of $\lambda$ and denoted by $\lambda_{t \text {-core }}$, which is actually independent of the ordering of removing $t$-hooks from $\lambda$ (see $[2,12]$ ). For $0 \leq i \leq t-1$, the $i$-th $t$-quotient of the partition $\lambda$ is defined to be the partition associated with the 01-sequence obtained by taking the subsequence $\left(z_{t j+i}\right)_{j \in \mathbb{Z}}$ of $z(\lambda)$, which is denoted by $\lambda^{i}$. For each positive integer $t$, we actually build a map

$$
\{\text { partitions }\} \rightarrow\{t \text {-core partitions }\} \times\{\text { partitions }\}^{t}
$$

which sends a partition $\lambda$ to $\left(\lambda_{t \text {-core }} ; \lambda^{0}, \lambda^{1}, \ldots, \lambda^{t-1}\right)$. This map is a bijection, called the Littlewood decomposition of partitions at $t$ [14]. In this paper, we always set that a partition $\lambda$ is identical with its image under the Littlewood decomposition, i.e., we always write $\lambda=\left(\lambda_{t \text {-core }} ; \lambda^{0}, \lambda^{1}, \ldots, \lambda^{t-1}\right)$. The Littlewood decomposition has the following two fundamental properties (see [14]):

$$
|\lambda|=\left|\lambda_{t-\text { core }}\right|+t\left(\left|\lambda^{0}\right|+\left|\lambda^{1}\right|+\cdots+\left|\lambda^{t-1}\right|\right)
$$

and

$$
\left\{h / t \mid h \in \mathcal{H}_{t}(\lambda)\right\}=\mathcal{H}\left(\lambda^{0}\right) \cup \mathcal{H}\left(\lambda^{1}\right) \cup \cdots \cup \mathcal{H}\left(\lambda^{t-1}\right) .
$$

Let $f_{\lambda}$ (resp. $f_{\lambda / \mu}$ ) be the number of standard Young tableaux of shape $\lambda$ (resp. $\lambda / \mu$ ) and $H_{\lambda}=\prod_{\square \in \lambda} h_{\square}$ be the product of all hook lengths of boxes in $\lambda$. For convenience set $f_{\emptyset}=1$ and $H_{\emptyset}=1$ for the empty partition $\emptyset$. It is well known that $($ see $[5,8,13,22])$

$$
f_{\lambda}=\frac{|\lambda| !}{H_{\lambda}} \quad \text { and } \quad \frac{1}{n !} \sum_{|\lambda|=n} f_{\lambda}^{2}=1 .
$$

The latter identity defines a measure on partitions of size $n$, called the Plancherel measure. Alternatively, we can see $f_{\lambda}$ as counting "walks" among Young diagrams up to the shape $\lambda$ (so-called hook walks, see $[6,7]$ ).

1.4. Polynomiality of Plancherel averages and modified hook walks. Expanding on a formula of Nekrasov and Okounkov on hook lengths [15], the second author conjectured [9] that

$$
P(n)=\frac{1}{n !} \sum_{|\lambda|=n} f_{\lambda}^{2} \sum_{\square \in \lambda} h_{\square}^{2 k}
$$

is always a polynomial of $n$ for any $k \in \mathbb{N}$. This conjecture was generalized and proved by Stanley [20] (see also [1], [4], [10] and [18]), and later generalized in [11]. Let $Q$ be a symmetric function in infinitely many variables and $E$ be a finite set with $n$ elements. The symbol $Q(x: x \in E)$ means that $n$ of the variables are substituted by $x$ for $x \in E$, and all other variables by 0 . 
Theorem 1.2 (Stanley [20]). Let $Q$ be a symmetric function in infinitely many variables. Then

$$
P(n)=\frac{1}{n !} \sum_{|\lambda|=n} f_{\lambda}^{2} Q\left(c_{\square}: \square \in \lambda\right)
$$

and

$$
P^{\prime}(n)=\frac{1}{n !} \sum_{|\lambda|=n} f_{\lambda}^{2} Q\left(h_{\square}^{2}: \square \in \lambda\right)
$$

are polynomials in $n$.

Olshanski [17] also proved the content case of Theorem 1.2.

Let $\lambda$ and $\mu$ be two partitions. We say that $\lambda \geq_{t} \mu$ if $\lambda$ could be obtained by adding some $t$-hooks to $\mu$. Then, the set of all partitions becomes a partially ordered set under this relation. If $\lambda \geq_{t} \mu$, define $F_{\lambda / \mu}$ to be the number of ways of removing $t$-hooks:

$$
\left.F_{\mu / \mu}:=1 \quad \text { and } \quad F_{\lambda / \mu}:=\sum_{\substack{\lambda \geq \lambda^{-} \geq_{t} \mu \\\left|\lambda / \lambda^{-}\right|=t}} F_{\lambda^{-} / \mu} \quad \text { (for } \lambda \neq \mu\right) .
$$

It is easy to see that $F_{\lambda / \mu}=\#\left\{\left(P_{0}, P_{1}, \ldots, P_{t-1}\right): P_{i}\right.$ is a Young tableau of shape $\lambda^{i} / \mu^{i}$, the union of entries in $P_{0}, P_{1}, \ldots, P_{t-1}$ are $\left.1,2, \ldots, \sum_{i=0}^{t-1}\left|\lambda^{i} / \mu^{i}\right|\right\}$. Hence,

$$
F_{\lambda / \mu}=\left(\begin{array}{c}
\sum_{i=0}^{t-1}\left|\lambda^{i} / \mu^{i}\right| \\
\left|\lambda^{0} / \mu^{0}\right|,\left|\lambda^{1} / \mu^{1}\right|, \ldots,\left|\lambda^{t-1} / \mu^{t-1}\right|
\end{array}\right) \prod_{i=0}^{t-1} f_{\lambda^{i} / \mu^{i}} .
$$

These $F_{\lambda / \mu}$ can be seen as counting hook walks distributed among the quotient partitions of $\lambda$. Let

$$
F_{\lambda}:=F_{\lambda / \lambda_{t \text {-core }}}=\left(\begin{array}{c}
n \\
\left|\lambda^{0}\right|,\left|\lambda^{1}\right|, \cdots,\left|\lambda^{t-1}\right|
\end{array}\right) \prod_{i=0}^{t-1} f_{\lambda^{i}}=n ! t^{n} G_{\lambda}
$$

where $n=\left|\lambda^{0}\right|+\left|\lambda^{1}\right|+\ldots+\left|\lambda^{t-1}\right|$ and

$$
G_{\lambda}:=\frac{1}{\prod_{h \in \mathcal{H}_{t}(\lambda)} h} .
$$

When $t=1$, then $F_{\lambda}=f_{\lambda}$ and $G_{\lambda}=1 / H_{\lambda}$. Also, when $\lambda$ is a $t$-core partition, we have $F_{\lambda}=G_{\lambda}=1$.

1.5. Difference operators and main result. Let $g$ be a function of partitions and $\lambda$ a partition. The difference operator $D$ for partitions was defined in [11] as

$$
D g(\lambda)=\sum_{\left|\lambda^{+} / \lambda\right|=1} g\left(\lambda^{+}\right)-g(\lambda) .
$$

In this paper, we introduce a generalization of $D$. For every integer $t$, let

$$
D_{t} g(\lambda)=\sum_{\substack{\lambda^{+} \geq t \lambda \\\left|\lambda^{+} / \lambda\right|=t}} g\left(\lambda^{+}\right)-g(\lambda)
$$

Accordingly, $D_{1}=D$.

The higher-order difference operators for $D_{t}$ are defined by induction:

$$
D_{t}^{0} g:=g \quad \text { and } \quad D_{t}^{k} g:=D_{t}\left(D_{t}^{k-1} g\right) \quad(k \geq 1) .
$$


These operators also fit in Stanley's theory of differential posets, although this is a language we will not use here [21]. The following theorem is our main result, which will be proved in Section 5 .

Theorem 1.3. Suppose that $t$ is a positive integer, $u^{\prime}, v^{\prime}, j_{u}, j^{\prime}{ }_{v}, k_{u}, k^{\prime}{ }_{v}$ are nonnegative integers and $\mu$ is a given t-core partition. Then, there exists some fixed $r \in \mathbb{N}$ such that

$$
D_{t}^{r}\left(G_{\lambda}\left(\prod_{u=1}^{u^{\prime}} \sum_{\substack{\square \in \lambda \\ h_{\square} \equiv \pm j_{u}(\bmod t)}} h_{\square}^{2 k_{u}}\right)\left(\prod_{v=1}^{v^{\prime}} \sum_{\substack{\square \in \lambda \\ c_{\square} \equiv j_{v}^{\prime}(\bmod t)}} c_{\square}^{k_{v}^{\prime}}\right)\right)=0
$$

for every partition $\lambda$ with $\lambda_{t \text {-core }}=\mu$. Moreover,

$$
P(n)=\sum_{\substack{\lambda_{t-c o r e}=\mu \\ \lambda / \mu \mid=n t}} F_{\lambda / \mu} G_{\lambda}\left(\prod_{u=1}^{u^{\prime}} \sum_{\substack{\square \in \lambda \\ h_{\square} \equiv \pm j_{u}(\bmod t)}} h_{\square}^{2 k_{u}}\right)\left(\prod_{v=1}^{v^{\prime}} \sum_{\substack{\square \in \lambda \\ c_{\square} \equiv j_{v}^{\prime}(\bmod t)}} c_{\square}^{k_{v}^{\prime}}\right)
$$

is a polynomial in $n$ ( $n$ is a nonnegative integer).

Let $Q_{1}$ and $Q_{2}$ be two symmetric functions. By Theorem 1.3, there exists some fixed $r \in \mathbb{N}$ such that

$$
D_{t}^{r}\left(G_{\lambda} Q_{1}\left(h_{\square}^{2}: \square \in \lambda\right) Q_{2}\left(c_{\square}: \square \in \lambda\right)\right)=0
$$

for every partition $\lambda$ with $\lambda_{t \text {-core }}=\mu$. Moreover,

$$
P(n)=\sum_{\substack{\lambda_{t-\text { core }}=\mu \\|\lambda / \mu|=n t}} F_{\lambda / \mu} G_{\lambda} Q_{1}\left(h_{\square}^{2}: \square \in \lambda\right) Q_{2}\left(c_{\square}: \square \in \lambda\right)
$$

is a polynomial of $n$. When $t=1, \mu=\emptyset$, we have (1.7) implies Theorem 1.2.

1.6. Specialisation for square case. We now focus on expressions of square sum of contents or hook lengths, and thereby obtain explicit results. Details of the proofs are given in Section 6. We start by considering individual partitions.

Theorem 1.4. Let $0 \leq k \leq t-1$. For every partition $\lambda$ with $\lambda_{t \text {-core }}=\emptyset$ we have

$$
\begin{aligned}
& \sum_{\substack{\square \in \lambda \\
h_{\square} \equiv k(\bmod t)}} h_{\square}^{2}+\sum_{\substack{\square \in \lambda \\
h_{\square} \equiv t-k(\bmod t)}} h_{\square}^{2}-\left(\sum_{\substack{\square \in \lambda \\
c_{\square} \equiv k(\bmod t)}} c_{\square}^{2}+\sum_{\substack{\square \in \lambda \\
c_{\square} \equiv t-k(\bmod t)}} c_{\square}^{2}\right) \\
= & 2 t^{2}\left(\sum_{i=0}^{t-1-k} n_{i} n_{i+k}+\sum_{i=0}^{k-1} n_{i} n_{i+t-k}\right),
\end{aligned}
$$

where $n_{i}=\left|\lambda^{i}\right|$.

By summing identity (1.8) over $0 \leq k \leq t-1$, we obtain the following well-known result (see [14]).

Corollary 1.5. For every partition $\lambda$ we have

$$
\sum_{\square \in \lambda} h_{\square}^{2}-\sum_{\square \in \lambda} c_{\square}^{2}=|\lambda|^{2} .
$$


Notice that the above identity can also be obtained from Theorem 1.4 with $t=1$.

We now turn to $t$-Plancherel averages, introducing $F_{\lambda / \mu}$ and $G_{\lambda}$ as weights in the previous results and summing over $\lambda$. The results are stated in two cases according to the divisibility of the hook lengths by $t$.

Theorem 1.6. Suppose that $\mu$ is a given $t$-core partition and $1 \leq k \leq t-1$. Then we have

$$
\begin{array}{r}
\sum_{\substack{\lambda t \text {-core }=\mu \\
|\lambda / \mu|=n t}} F_{\lambda / \mu} G_{\lambda}\left(\sum_{\substack{\square \in \lambda \\
h_{\square} \equiv k(\bmod t)}} h_{\square}^{2}+\sum_{\substack{\square \in \lambda \\
h_{\square} \equiv t-k(\bmod t)}} h_{\square}^{2}\right) \\
=6 t\left(\begin{array}{c}
n \\
2
\end{array}\right)+ \\
+\left(\sum_{\substack{\square \in \mu \\
h_{\square} \equiv k(\bmod t)}} h_{\square}^{2}+\sum_{\substack{\square \in \mu \\
h_{\square} \equiv t-k(\bmod t)}} h_{\square}^{2}\right),
\end{array}
$$

where $\mu(k)=\{h \in \mathcal{H}(\mu) \mid h \equiv k(\bmod t)\}$, viewed as a multiset. In particular, let $\mu=\emptyset$. Then we have

$$
\begin{aligned}
& \sum_{\substack{\lambda_{t-c o r e}=\emptyset \\
|\lambda|=n t}} F_{\lambda} G_{\lambda}\left(\sum_{\substack{\square \in \lambda \\
h_{\square} \equiv k(\bmod t)}} h_{\square}^{2}+\sum_{\substack{\square \in \lambda \\
h_{\square} \equiv t-k(\bmod t)}} h_{\square}^{2}\right) \\
= & 6 t\left(\begin{array}{l}
n \\
2
\end{array}\right)+2 k(t-k) n .
\end{aligned}
$$

Theorem 1.7. Suppose that $\mu$ is a given t-core partition. Then we have

$$
\sum_{\substack{\lambda t \text { core }=\mu \\
|\lambda / \mu|=n t}} F_{\lambda / \mu} G_{\lambda} \sum_{\substack{\square \in \lambda \\
h_{\square} \equiv 0(\bmod t)}} h_{\square}^{2}=n t^{2}+3 t\left(\begin{array}{l}
n \\
2
\end{array}\right) .
$$

By Theorems 1.6 and 1.7 we obtain the following result.

Corollary 1.8. Suppose that $\mu$ is a given t-core partition. Then

$$
\sum_{\substack{\lambda_{t} \text {-core }=\mu \\|\lambda / \mu|=n t}} F_{\lambda / \mu} G_{\lambda} \sum_{\square \in \lambda} h_{\square}^{2}=\frac{3 t^{2} n^{2}}{2}+\frac{n t\left(t^{2}-3 t-1+24|\mu|\right)}{6}+\sum_{\square \in \mu} h_{\square}^{2} .
$$

In particular, let $\mu=\emptyset$. Then we have

$$
\sum_{\substack{\lambda_{t-\text { core }=\emptyset}|\lambda|=n t \\|\lambda|}} F_{\lambda} G_{\lambda} \sum_{\square \in \lambda} h_{\square}^{2}=\frac{3 t^{2} n^{2}}{2}+\frac{n t\left(t^{2}-3 t-1\right)}{6} .
$$

Similar results for contents can also be obtained.

Theorem 1.9. Let $0 \leq k \leq t-1$. We have

$$
\sum_{\substack{\lambda_{t-\text { core }}=\emptyset \\
|\lambda|=n t}} F_{\lambda} G_{\lambda} \sum_{\substack{\square \in \lambda \\
c_{\square} \equiv k(\bmod t)}} c_{\square}^{2}=t\left(\begin{array}{l}
n \\
2
\end{array}\right)+k(t-k) n .
$$


Theorem 1.9 implies the following identity.

$$
\sum_{\substack{\lambda t-\text { core }=\emptyset \\
|\lambda|=n t}} F_{\lambda} G_{\lambda} \sum_{\square \in \lambda} c_{\square}^{2}=t^{2}\left(\begin{array}{l}
n \\
2
\end{array}\right)+\frac{\left(t^{3}-t\right) n}{6} .
$$

In fact we will derive a more general result in Theorem 6.3 by replacing $\emptyset$ by a general $t$-core partition.

\section{Partitions And $t$-Difference operator}

We now turn our attention to preliminary lemmas on the $t$-difference operators $D_{t}^{k}$, and in particular a theorem outlining how polynomiality of $t$-Plancherel averages can be deduced from the vanishing of $D_{t}^{k}$ for large enough $k$. It is known [11] that

$$
D\left(\frac{1}{H_{\lambda}}\right)=0
$$

We establish a similar result for the $t$-difference operator.

Lemma 2.1. Suppose that $\lambda$ is a partition. Then

$$
D_{t}\left(G_{\lambda}\right)=0 \text {. }
$$

In other words,

$$
G_{\lambda}=\sum_{\substack{\lambda^{+} \geq{ }_{t} \lambda \\\left|\lambda^{+} / \lambda\right|=t}} G_{\lambda^{+}}
$$

Proof. Suppose that

$$
\lambda=\left(\lambda_{t \text {-core }} ; \lambda^{0}, \lambda^{1}, \ldots, \lambda^{i-1}, \lambda^{i}, \lambda^{i+1}, \ldots, \lambda^{t-1}\right)
$$

and

$$
\lambda^{+}=\left(\lambda_{t \text {-core }} ; \lambda^{0}, \lambda^{1}, \ldots, \lambda^{i-1},\left(\lambda^{i}\right)^{+}, \lambda^{i+1}, \ldots, \lambda^{t-1}\right)
$$

where $\left|\left(\lambda^{i}\right)^{+} / \lambda^{i}\right|=1$. Then, by the definition of $G_{\lambda}$ we have

$$
\frac{G_{\lambda^{+}}}{G_{\lambda}}=\frac{H_{\lambda^{i}}}{t H_{\left(\lambda^{i}\right)^{+}}} \text {. }
$$

By (2.1) we have

$$
\sum_{\left|\left(\lambda^{i}\right)^{+} / \lambda^{i}\right|=1} \frac{G_{\left(\lambda_{t-\mathrm{core}} ; \lambda^{0}, \lambda^{1}, \ldots, \lambda^{i-1},\left(\lambda^{i}\right)^{+}, \lambda^{i+1}, \ldots, \lambda^{t-1}\right)}}{G_{\lambda}}=\frac{1}{t} .
$$

Summing the above equality over $0 \leq i \leq t-1$, we prove our claim.

We now prove a commutation relation between the averaging over partitions of a given weight and the $D_{t}$ operator.

Lemma 2.2. Suppose that $\mu$ is a given partition and $g$ is a function of partitions. For every $n \in \mathbb{N}$, let

$$
P_{g}(n):=\sum_{\substack{\lambda \geq t \mu \\ \lambda \lambda / \mu \mid=n t}} F_{\lambda / \mu} g(\lambda)
$$

Then

$$
P_{g}(n+1)-P_{g}(n)=P_{D_{t} g}(n) .
$$


Proof. The proof is straightforward and follows from expanding definitions:

$$
\begin{aligned}
P_{g}(n+1)-P_{g}(n) & =\sum_{\substack{\nu \geq t \mu \\
|\nu / \mu|=(n+1) t}} F_{\nu / \mu} g(\nu)-\sum_{\substack{\lambda \geq t \mu \\
|\lambda / \mu|=n t \\
\mid \mu / \mu}} F_{\lambda / \mu} g(\lambda) \\
& =\sum_{\substack{\nu \geq_{t} \mu \\
|\nu / \mu|=(n+1) t}} \sum_{\substack{\nu \geq_{t} \nu^{-} \geq_{t} \mu \\
\left|\nu / \nu^{-}\right|=t}} F_{\nu^{-} / \mu} g(\nu)-\sum_{\substack{\lambda \geq_{t} \mu \\
|\lambda / \mu|=n t}} F_{\lambda / \mu} g(\lambda) \\
& =\sum_{\substack{\lambda \geq t \mu \\
|\lambda / \mu|=n t}} F_{\lambda / \mu}\left(\sum_{\substack{\lambda^{+} \geq_{t} \lambda \\
\left|\lambda^{+} / \lambda\right|=t}} g\left(\lambda^{+}\right)-g(\lambda)\right) \\
& =P_{D_{t} g}(n) .
\end{aligned}
$$

Example. Let $g(\lambda)=G_{\lambda}$. Then $D_{t} g(\lambda)=0$ by Lemma 2.1, which means that $P_{D_{t} g}(n)=0$. Consequently, $P_{g}(n+1)=P_{g}(n)=\cdots=P_{g}(0)=G_{\mu}$, or

$$
\sum_{\substack{\lambda \geq t \mu \\|\lambda / \mu|=n t}} F_{\lambda / \mu} G_{\lambda}=G_{\mu} .
$$

Recall that when $\mu$ is a $t$-core partition, we have $G_{\mu}=1$. This implies

$$
\sum_{\substack{\lambda t-\text { core }=\mu \\|\lambda / \mu|=n t}} F_{\lambda / \mu} G_{\lambda}=1
$$

In particular, let $\mu=\emptyset$, we obtain a generalization of the second identity of (1.1):

$$
\sum_{\substack{\lambda t-\text { core }=\emptyset \\|\lambda|=n t}} F_{\lambda} G_{\lambda}=1
$$

or equivalently,

$$
\sum_{\left|\lambda^{0}\right|+\left|\lambda^{1}\right|+\cdots+\left|\lambda^{t-1}\right|=n}\left(\begin{array}{c}
n \\
\left|\lambda^{0}\right|,\left|\lambda^{1}\right|, \cdots,\left|\lambda^{t-1}\right|
\end{array}\right) \prod_{i=0}^{t-1} \frac{f_{\lambda^{i}}^{2}}{\left|\lambda^{i}\right| !}=t^{n} .
$$

Formulas such as this one can be used to create new measures on partitions, called t-Plancherel.

Recall that the higher-order difference operators for $D_{t}$ are defined by induction $D_{t}^{0} g:=g$ and $D_{t}^{k} g:=D_{t}\left(D_{t}^{k-1} g\right)(k \geq 1)$. We write $D_{t} g(\nu):=\left.D_{t} g(\lambda)\right|_{\lambda=\nu}$ for a fixed partition $\nu$.

Theorem 2.3. Let $g$ be a function of partitions and $\mu$ be a given partition. Then,

$$
P_{g}(n)=\sum_{\substack{\lambda \geq t \mu \\
|\lambda / \mu|=n t}} F_{\lambda / \mu} g(\lambda)=\sum_{k=0}^{n}\left(\begin{array}{l}
n \\
k
\end{array}\right) D_{t}^{k} g(\mu)
$$

and

$$
D_{t}^{n} g(\mu)=\sum_{k=0}^{n}(-1)^{n+k}\left(\begin{array}{l}
n \\
k
\end{array}\right) P_{g}(k) .
$$

In particular, if there exists some positive integer $r$ such that $D_{t}^{r} g(\lambda)=0$ for every partition $\lambda \geq_{t} \mu$, then $P_{g}(n)$ is a polynomial in $n$. 
Proof. Identity (2.6) will be proved by induction. The case $n=0$ is obvious. Assume that (2.6) is true for some nonnegative integer $n$. By Lemma 2.2 we obtain

$$
\begin{aligned}
P_{g}(n+1) & =P_{g}(n)+P_{D_{t} g}(n) \\
& =\sum_{k=0}^{n}\left(\begin{array}{l}
n \\
k
\end{array}\right) D_{t}^{k} g(\mu)+\sum_{k=0}^{n}\left(\begin{array}{l}
n \\
k
\end{array}\right) D_{t}^{k+1} g(\mu) \\
& =\sum_{k=0}^{n+1}\left(\begin{array}{c}
n+1 \\
k
\end{array}\right) D_{t}^{k} g(\mu) .
\end{aligned}
$$

Identity (2.7) follows from the famous Möbius inversion formula [19].

\section{Corners of PARTitions}

Now we introduce the concept of corners in a partition. For a partition $\lambda$, the outer corners (see $[3,11]$ ) are the boxes which can be removed in such a way that after removal the resulting diagram is still a Young one. The coordinates of outer corners are denoted by $\left(\alpha_{1}, \beta_{1}\right), \ldots,\left(\alpha_{m}, \beta_{m}\right)$ such that $\alpha_{1}>\alpha_{2}>\cdots>\alpha_{m}$. Let $y_{j}:=\beta_{j}-\alpha_{j}(1 \leq j \leq m)$ be the contents of outer corners. Set $\alpha_{m+1}=$ $\beta_{0}=0$. The inner corners of $\lambda$ are defined to be the boxes whose coordinates are $\left(\alpha_{1}, \beta_{0}\right),\left(\alpha_{2}, \beta_{1}\right), \ldots,\left(\alpha_{m+1}, \beta_{m}\right)$. Let $x_{i}=\beta_{i}-\alpha_{i+1}$ be the contents of the inner corners for $0 \leq i \leq m$.

Example. For $\lambda=(6,3,2,2)$ (see Figure 1) we have $m=3,\left(\alpha_{1}, \beta_{1}\right)=(4,2)$, $\left(\alpha_{2}, \beta_{2}\right)=(2,3)$ and $\left(\alpha_{3}, \beta_{3}\right)=(1,6)$. Therefore $\left(x_{0}, x_{1}, x_{2}, x_{3}\right)=(-4,0,2,6)$ and $\left(y_{1}, y_{2}, y_{3}\right)=(-2,1,5)$. Also note that $\sum_{0 \leq i \leq 3} x_{i}-\sum_{1 \leq j \leq 3} y_{j}=0$ and $\sum_{0 \leq i \leq 3} x_{i}{ }^{2}-\sum_{1 \leq j \leq 3} y_{j}{ }^{2}=26=2|\lambda|$.

Let $\lambda$ be a partition and $x_{i}, y_{j}$ be the quantities associated to $\lambda$ defined above. Define (see [11])

$$
q_{k}(\lambda):=\sum_{0 \leq i \leq m} x_{i}^{k}-\sum_{1 \leq j \leq m} y_{j}^{k}
$$

for each integer $k$, and

$$
q_{\nu}(\lambda):=\prod_{k=1}^{\ell} q_{\nu_{k}}(\lambda)
$$

for each partition $\nu=\left(\nu_{1}, \nu_{2}, \ldots, \nu_{\ell}\right){ }^{2}$

We need the following results developed in [11] (see (6.3), (6.6), Theorem 6.1 and Lemma 6.4 in [11] respectively).

Theorem 3.1. Let $\lambda^{i+}=\lambda \cup\left\{\square_{i}\right\}$ with $c_{\square_{i}}=x_{i}$ for $0 \leq i \leq m$.

(1) For $k=0,1,2$, we have

$$
q_{0}(\lambda)=1, \quad q_{1}(\lambda)=0 \quad \text { and } \quad q_{2}(\lambda)=2|\lambda| .
$$

(2) Suppose that $k$ is a nonnegative integer. Then we have

$$
q_{k}\left(\lambda^{i+}\right)-q_{k}(\lambda)=\sum_{1 \leq j \leq k / 2} 2\left(\begin{array}{c}
k \\
2 j
\end{array}\right) x_{i}^{k-2 j}
$$

\footnotetext{
${ }^{2}$ We need to be careful here: in general, $q_{\nu}(\lambda)=\prod_{i} q_{\nu_{i}}(\lambda) \neq \prod_{i} q_{\nu^{i}}(\lambda)$, which was used in Theorem 3.4 and commented on in footnote 3. Writing $q_{\nu}$ allows for a convenient access to $|\nu|$.
} 
(3) Let $\nu$ be a partition. Then, there exist some $b_{\delta} \in \mathbb{Q}$ such that

$$
D\left(\frac{q_{\nu}(\lambda)}{H_{\lambda}}\right)=\sum_{|\delta| \leq|\nu|-2} b_{\delta} \frac{q_{\delta}(\lambda)}{H_{\lambda}}
$$

for every partition $\lambda$.

(4) Let $k$ be a nonnegative integer. Then there exist some $b_{\delta} \in \mathbb{Q}$ such that

$$
\sum_{0 \leq i \leq m} \frac{H_{\lambda}}{H_{\lambda^{i+}}} x_{i}^{k}=\sum_{|\delta| \leq k} b_{\delta} q_{\delta}(\lambda)
$$

for every partition $\lambda$.

The next result is easy to prove by Theorem 3.1(4).

Theorem 3.2. Let $\lambda^{i+}=\lambda \cup\left\{\square_{i}\right\}$ with $c_{\square_{i}}=x_{i}$ for $0 \leq i \leq m$. Then,

$$
\sum_{0 \leq i \leq m} \frac{H_{\lambda}}{H_{\lambda^{i+}}} x_{i}=0
$$

and

for every partition $\lambda$.

$$
\sum_{0 \leq i \leq m} \frac{H_{\lambda}}{H_{\lambda^{i+}}} x_{i}^{2}=|\lambda|
$$

We obtain the following result, which is a generalization of Theorem 3.1(3).

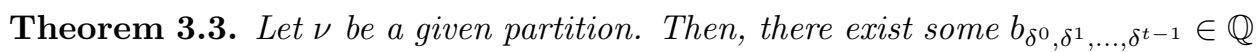
such that

$$
D_{t}\left(G_{\lambda} \prod_{i=0}^{t-1} q_{\nu^{i}}\left(\lambda^{i}\right)\right)=\sum_{(*)} b_{\delta^{0}, \delta^{1}, \ldots, \delta^{t-1}} G_{\lambda} \prod_{i=0}^{t-1} q_{\delta^{i}}\left(\lambda^{i}\right)
$$

for every partition $\lambda$, where $(*)$ ranges over all partitions $\delta^{0}, \delta^{1}, \ldots, \delta^{t-1}$ such that $\sum_{i=0}^{t-1}\left|\delta^{i}\right| \leq \sum_{i=0}^{t-1}\left|\nu^{i}\right|-2$. Notice that $b_{\delta^{0}, \delta^{1}, \ldots, \delta^{t-1}}$ is independent of $\lambda$.

Furthermore, if $r \geq \frac{1}{2} \sum_{i=0}^{t-1}\left|\nu^{i}\right|+1$, then

$$
D_{t}^{r}\left(G_{\lambda} \prod_{i=0}^{t-1} q_{\nu^{i}}\left(\lambda^{i}\right)\right)=0
$$

for every partition $\lambda$.

Proof. We have

$$
\begin{aligned}
& D_{t}\left(G_{\lambda} \prod_{i=0}^{t-1} q_{\nu^{i}}\left(\lambda^{i}\right)\right) \\
= & \sum_{\substack{\lambda^{+} t_{t} \lambda \\
\left|\lambda^{+} / \lambda\right|=t}} G_{\lambda^{+}} \prod_{i=0}^{t-1} q_{\nu^{i}}\left(\left(\lambda^{+}\right)^{i}\right)-G_{\lambda} \prod_{i=0}^{t-1} q_{\nu^{i}}\left(\lambda^{i}\right) \\
= & G_{\lambda} \sum_{\substack{\lambda^{+} \geq t \lambda \\
\left|\lambda^{+} / \lambda\right|=t}} \frac{G_{\lambda^{+}}}{G_{\lambda}}\left(\prod_{i=0}^{t-1} q_{\nu^{i}}\left(\left(\lambda^{+}\right)^{i}\right)-\prod_{i=0}^{t-1} q_{\nu^{i}}\left(\lambda^{i}\right)\right)
\end{aligned}
$$




$$
\begin{aligned}
& =\frac{1}{t} G_{\lambda} \sum_{j=0}^{t-1} \prod_{\substack{0 \leq i \leq t-1 \\
i \neq j}} q_{\nu^{i}}\left(\lambda^{i}\right) \sum_{\left|\left(\lambda^{j}\right)^{+} / \lambda^{j}\right|=1} \frac{H_{\lambda^{j}}}{H_{\left(\lambda^{j}\right)^{+}}}\left(q_{\nu^{j}}\left(\left(\lambda^{j}\right)^{+}\right)-q_{\nu^{j}}\left(\lambda^{j}\right)\right) \\
& =\frac{1}{t} G_{\lambda} \sum_{j=0}^{t-1} \prod_{\substack{0 \leq i \leq t-1 \\
i \neq j}} q_{\nu^{i}}\left(\lambda^{i}\right) H_{\lambda^{j}} D\left(\frac{q_{\nu^{j}}\left(\lambda^{j}\right)}{H_{\lambda^{j}}}\right) \\
& =\sum_{(*)} b_{\delta^{0}, \delta^{1}, \ldots, \delta^{t-1}} G_{\lambda} \prod_{i=0}^{t-1} q_{\delta^{i}}\left(\lambda^{i}\right)
\end{aligned}
$$

for some $b_{\delta^{0}, \delta^{1}, \ldots, \delta^{t-1}} \in \mathbb{Q}$, where $(*)$ ranges over all partitions $\delta^{0}, \delta^{1}, \ldots, \delta^{t-1}$ such that $\sum_{i=0}^{t-1}\left|\delta^{i}\right| \leq \sum_{i=0}^{t-1}\left|\nu^{i}\right|-2$. The last equality is due to Theorem 3.1(3).

Definition 3.1. Let $\mu$ be a $t$-core partition. A function $g$ of partitions is called $\mu$-admissible, if there exist some functions of partitions $\eta_{s}$ (here $\eta_{s}$ depends on $t, g, i, \mu)$ such that $g\left(\lambda^{+}\right)-g(\lambda)=\sum_{s=0}^{s^{\prime}} \eta_{s}(\lambda) c_{\square_{i}}^{s}$ is a polynomial of $c_{\square_{i}}$ for every pair of partitions

$$
\lambda=\left(\mu ; \lambda^{0}, \lambda^{1}, \ldots, \lambda^{i-1}, \lambda^{i}, \lambda^{i+1}, \ldots, \lambda^{t-1}\right)
$$

and

$$
\lambda^{+}=\left(\mu ; \lambda^{0}, \lambda^{1}, \ldots, \lambda^{i-1}, \lambda^{i} \cup\left\{\square_{i}\right\}, \lambda^{i+1}, \ldots, \lambda^{t-1}\right),
$$

where each coefficient $\eta_{s}(\lambda)$ is a linear combination of some $\prod_{j=0}^{t-1} q_{\tau^{j}}\left(\lambda^{j}\right)$ for some partition $\tau,{ }^{3}$ and for every partition $\lambda$ with $\lambda_{t \text {-core }}=\mu$.

By Theorem 3.1(2), $\lambda \mapsto \prod_{i=0}^{t-1} q_{\nu^{i}}\left(\lambda^{i}\right)$ is $\mu$-admissible for any partition $\nu$ and any $t$-core partition $\mu$. As can be seen in Section 5, the following two functions of partitions

$$
g(\lambda)=\sum_{\substack{\square \in \lambda \\ h_{\square} \equiv \pm j(\bmod t)}} h_{\square}^{2 k}
$$

and

$$
g(\lambda)=\sum_{\substack{\square \in \lambda \\ c_{\square} \equiv j(\bmod t)}} c_{\square}^{k}
$$

are $\mu$-admissible for any $t$-core partition $\mu$, any nonnegative integer $k$, and $0 \leq j \leq$ $t-1$. This is a consequence of Lemmas 5.2 and 5.4.

Theorem 3.4. Let $t$ be a given integer, $\nu$ be a partition, and $\mu$ be a t-core partition. Suppose that $g_{1}, g_{2}, \ldots, g_{v}$ are $\mu$-admissible functions of partitions. Then, there exists some $r \in \mathbb{N}$ such that

$$
D_{t}^{r}\left(G_{\lambda} \prod_{u=1}^{v} g_{u}(\lambda) \prod_{i=0}^{t-1} q_{\nu^{i}}\left(\lambda^{i}\right)\right)=0
$$

\footnotetext{
${ }^{3}$ We really need here a $t$-tuple of partitions. We decide to reuse the quotient notation for this purpose. Different $\tau$ with the same $t$-core would give the same tuple, but the surjectivity is inconsequential here.
} 
for every partition $\lambda$ with $\lambda_{t \text {-core }}=\mu$. Furthermore, by Theorem 2.3,

$$
P(n)=\sum_{\substack{\lambda t-c o r e=\mu \\|\lambda / \mu|=n t}} F_{\lambda / \mu} G_{\lambda} \prod_{u=1}^{v} g_{u}(\lambda)
$$

is a polynomial in $n$.

Proof of Theorem 3.4. Notice that this theorem is true for $v=0$ by Theorem 3.3.

We will prove it by induction. First, we have

$$
\begin{aligned}
& D_{t}\left(G_{\lambda} \prod_{u=1}^{v} g_{u}(\lambda) \prod_{i=0}^{t-1} q_{\nu^{i}}\left(\lambda^{i}\right)\right) \\
= & G_{\lambda} \sum_{\substack{\lambda^{+} \geq t \lambda \\
\left|\lambda^{+} / \lambda\right|=t}} \frac{G_{\lambda^{+}}}{G_{\lambda}}\left(\prod_{u=1}^{v} g_{u}\left(\lambda^{+}\right) \prod_{i=0}^{t-1} q_{\nu^{i}}\left(\left(\lambda^{+}\right)^{i}\right)-\prod_{u=1}^{v} g_{u}(\lambda) \prod_{i=0}^{t-1} q_{\nu^{i}}\left(\lambda^{i}\right)\right) \\
= & G_{\lambda} \sum_{\substack{\lambda^{+} \geq t \lambda \\
\left|\lambda^{+} / \lambda\right|=t}} \frac{G_{\lambda^{+}}}{G_{\lambda}}(A \cdot \Delta B+B \cdot \Delta A+\Delta A \cdot \Delta B),
\end{aligned}
$$

where

$$
\begin{aligned}
A & =\prod_{u=1}^{v} g_{u}(\lambda), \\
\Delta A & =\prod_{u=1}^{v} g_{u}\left(\lambda^{+}\right)-\prod_{u=1}^{v} g_{u}(\lambda), \\
B & =\prod_{i=0}^{t-1} q_{\nu^{i}}\left(\lambda^{i}\right), \\
\Delta B & =\prod_{i=0}^{t-1} q_{\nu^{i}}\left(\left(\lambda^{+}\right)^{i}\right)-\prod_{i=0}^{t-1} q_{\nu^{i}}\left(\lambda^{i}\right) .
\end{aligned}
$$

Next,

$$
\begin{aligned}
& G_{\lambda} \sum_{\substack{\lambda^{+} \geq t \lambda \\
\left|\lambda^{+} / \lambda\right|=t}} \frac{G_{\lambda^{+}}}{G_{\lambda}}(A \cdot \Delta B) \\
& =\frac{1}{t} G_{\lambda} \prod_{u=1}^{v} g_{u}(\lambda) \sum_{j=0}^{t-1}\left(\prod_{\substack{0 \leq i \leq t-1 \\
i \neq j}} q_{\nu^{i}}\left(\lambda^{i}\right)\right) H_{\lambda^{j}} D\left(\frac{q_{\nu^{j}}\left(\lambda^{j}\right)}{H_{\lambda^{j}}}\right), \\
& G_{\lambda} \sum_{\substack{\lambda^{+} \geq t \lambda \\
\left|\lambda^{+} / \lambda\right|=t}} \frac{G_{\lambda^{+}}}{G_{\lambda}}(B \cdot \Delta A) \\
& =\frac{1}{t} G_{\lambda} \prod_{i=0}^{t-1} q_{\nu^{i}}\left(\lambda^{i}\right) \sum_{j=0}^{t-1} \sum_{\left|\left(\lambda^{j}\right)^{+} / \lambda^{j}\right|=1} \frac{H_{\lambda^{j}}}{H_{\left(\lambda^{j}\right)^{+}}}
\end{aligned}
$$




$$
\begin{aligned}
& \left(\prod_{u=1}^{v} g_{u}\left(\left(\mu ; \lambda^{0}, \ldots,\left(\lambda^{j}\right)^{+}, \ldots, \lambda^{t-1}\right)\right)-\prod_{u=1}^{v} g_{u}(\lambda)\right), \\
G_{\lambda} & \sum_{\substack{\lambda^{+} \geq t \lambda \\
\left|\lambda^{+} / \lambda\right|=t}} \frac{G_{\lambda^{+}}}{G_{\lambda}}(\Delta A \cdot \Delta B) \\
= & \frac{1}{t} G_{\lambda} \sum_{j=0}^{t-1} \sum_{\left|\left(\lambda^{j}\right)^{+} / \lambda^{j}\right|=1} \frac{H_{\lambda^{j}}}{H_{\left(\lambda^{j}\right)^{+}}}\left(\prod_{\substack{0 \leq i \leq t-1 \\
i \neq j}} q_{\nu^{i}}\left(\lambda^{i}\right)\right)\left(q_{\nu^{j}}\left(\left(\lambda^{j}\right)^{+}\right)-q_{\nu^{j}}\left(\lambda^{j}\right)\right) \\
& \left(\prod_{u=1}^{v} g_{u}\left(\left(\mu ; \lambda^{0}, \ldots,\left(\lambda^{j}\right)^{+}, \ldots, \lambda^{t-1}\right)\right)-\prod_{u=1}^{v} g_{u}(\lambda)\right) .
\end{aligned}
$$

By Theorems 3.1 and 3.3 each of the last three terms could be written as a linear combination of some $G_{\lambda} \prod_{u^{\prime}=1}^{v^{\prime}} g^{\prime}{ }_{u^{\prime}}(\lambda) \prod_{i=0}^{t-1} q_{\left(\nu^{\prime}\right)^{i}}\left(\lambda^{i}\right)$ where, either $v^{\prime}=v$ and simultaneously $\sum_{i=0}^{t-1}\left(\nu^{\prime}\right)^{i} \leq \sum_{i=0}^{t-1} \nu^{i}-2$, or $v^{\prime}<v$. Then, by Theorem 3.3 and induction we prove the claim.

\section{Further PRoperties of LitTlewood DECOMPosition}

In this section, we always assume that $\mu$ is a given $t$-core partition with 01sequence $z(\mu)=\left(z_{\mu, j}\right)_{j \in \mathbb{Z}}$. For $0 \leq i \leq t-1$, we have $\mu^{i}=\emptyset$. Thus, $\mu^{i}$ has a 01 -sequence equal to $\cdots 0000 \mid 11111 \cdots$, which means that $z_{\mu^{i}, j}=0$ for $j<0$ and $z_{\mu^{i}, j}=1$ for $j \geq 0$. Let

$$
b_{i}:=b_{i}(\mu)=\min \left\{j \in \mathbb{Z}: j \equiv i(\bmod t), z_{\mu, j}=1\right\} .
$$

Then, there exist some $d_{i} \in \mathbb{Z}$ such that $b_{i}=t d_{i}+i$ for $0 \leq i \leq t-1$. Therefore, $z_{\mu, t j+i}=0$ for $j<d_{i}$ and $z_{\mu, t j+i}=1$ for $j \geq d_{i}$.

Example. The 01-sequence of the 3-core partition $(5,3,1,1)$ is

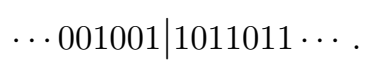

Therefore, $b_{0}=0, b_{1}=7, b_{2}=-4, d_{0}=0, d_{1}=2$, and $d_{2}=-2$. Notice that $d_{0}+d_{1}+d_{2}=0$.

Let

$$
B_{k}=\{(i, i+k): 0 \leq i \leq t-1-k\} \bigcup\{(i, i+t-k): 0 \leq i \leq k-1\},
$$

viewed as multisets for $1 \leq k \leq t-1$ (i.e., if $k$ equals $t-k$, we keep two copies). We have

$$
\sum_{(i, j) \in B_{k}}(j-i)^{2}=t k(t-k)
$$

Lemma 4.1. Suppose that $\mu$ is a given $t$-core partition and let $b_{i}$ and $d_{i}$ be defined as above. Then

$$
\sum_{i=0}^{t-1} d_{i}=0 .
$$


Proof. Let $0 \leq i \leq t-1$. First, the fact that $\mu$ is a $t$-core partition implies that $z_{\mu, t j+i}=0$ for $j<d_{i}$ and $z_{\mu, t j+i}=1$ for $j \geq d_{i}$. By the definition of 01-sequence we have $\#\left\{z_{\mu, j}=0: j \geq 0\right\}=\#\left\{z_{\mu, j}=1: j<0\right\}$. Thus,

$$
\sum_{i=0}^{t-1} d_{i}=\#\left\{z_{\mu, j}=0: j \geq 0\right\}-\#\left\{z_{\mu, j}=1: j<0\right\}=0 .
$$

For every partition $\lambda$ and $0 \leq k \leq t-1$, recall that $\lambda(k)=\left\{h_{\square} \equiv k(\bmod t)\right.$ : $\square \in \lambda\}$, viewed as a multiset.

Lemma 4.2. Suppose that $\mu$ is a given $t$-core partition and $1 \leq k \leq t-1$. Then, $|\mu(0)|=0$ and

$$
|\mu(k)|+|\mu(t-k)|=\sum_{(i, j) \in B_{k}}\left(\begin{array}{c}
d_{i}-d_{j} \\
2
\end{array}\right) .
$$

Proof. First, $|\mu(0)|=0$ since $\mu$ is a $t$-core partition. By Lemma 1.1 we have

$$
|\mu(k)|=\#\left\{\left(i^{\prime}, j^{\prime}\right): i^{\prime}<j^{\prime}, j^{\prime}-i^{\prime} \equiv k(\bmod t), z_{\mu, i^{\prime}}=1, z_{\mu, j^{\prime}}=0\right\} .
$$

Now we just need to consider the right hand side of the above identity. Notice that for $0 \leq i \leq t-1$ we have $z_{\mu, t j+i}=0$ for $j<d_{i}$ and $z_{\mu, t j+i}=1$ for $j \geq d_{i}$. Then, for each $(i, j) \in B_{k}$, we have

$$
\begin{aligned}
& \#\left\{\left(i^{\prime}, j^{\prime}\right): i^{\prime} \equiv i(\bmod t), j^{\prime} \equiv j(\bmod t), i^{\prime}<j^{\prime}, z_{\mu, i^{\prime}}=1, z_{\mu, j^{\prime}}=0\right\} \\
& +\#\left\{\left(i^{\prime}, j^{\prime}\right): i^{\prime} \equiv j(\bmod t), j^{\prime} \equiv i(\bmod t), i^{\prime}<j^{\prime}, z_{\mu, i^{\prime}}=1, z_{\mu, j^{\prime}}=0\right\} \\
& =1+2+\cdots+\left(d_{i}-d_{j}-1\right)=\left(\begin{array}{c}
d_{i}-d_{j} \\
2
\end{array}\right)=\left(\begin{array}{c}
d_{j}-d_{i}+1 \\
2
\end{array}\right)
\end{aligned}
$$

whenever $d_{i}>d_{j}$ or $d_{i} \leq d_{j}$. This means that

$$
|\mu(k)|+|\mu(t-k)|=\sum_{(i, j) \in B_{k}}\left(\begin{array}{c}
d_{i}-d_{j} \\
2
\end{array}\right) .
$$

By Lemmas 4.1 and 4.2 we obtain

$$
\begin{aligned}
\sum_{(i, j) \in B_{k}}(2 j-2 i)\left(d_{i}-d_{j}\right) & =t \sum_{(i, j) \in B_{k}}\left(d_{i}-d_{j}\right), \\
t \sum_{0 \leq i \leq t-1} d_{i}^{2} & =\sum_{0 \leq i<j \leq t-1}\left(d_{i}-d_{j}\right)^{2}, \\
-2 \sum_{0 \leq i \leq t-1} i d_{i} & =\sum_{0 \leq i<j \leq t-1}\left(d_{i}-d_{j}\right),
\end{aligned}
$$

and

$$
\begin{aligned}
|\mu| & =\sum_{0 \leq i<j \leq t-1}\left(\begin{array}{c}
d_{i}-d_{j} \\
2
\end{array}\right) \\
& =\frac{t}{2} \sum_{0 \leq i \leq t-1} d_{i}^{2}+\sum_{0 \leq i \leq t-1} i d_{i}
\end{aligned}
$$




$$
=\frac{1}{2 t^{2}} \sum_{0 \leq i<j \leq t-1}\left(\left(b_{i}-b_{j}\right)^{2}-(i-j)^{2}\right) .
$$

Lemma 4.3. Suppose that $\mu$ is a given $t$-core partition and $0 \leq k \leq t-1$. Let $\lambda$ be a partition with $\lambda_{\text {-core }}=\mu$ and $|\lambda|=|\mu|+n t$. Then,

$$
|\lambda(k)|+|\lambda(t-k)|-|\mu(k)|-|\mu(t-k)|=2 n .
$$

Proof. Adding a $t$-hook to a partition $\lambda$ means that we change some $\left(z_{i}=0\right.$, $\left.z_{i+t}=1\right)$ to $\left(z_{i}=1, z_{i+t}=0\right)$ in the 01-sequence of $\lambda$. Then, the lemma follows directly from Lemma 1.1 and induction on the size of $\lambda$.

\section{Contents, Hook Lengths and $t$-Difference operators}

By the construction of 01-sequences and Lemma 1.1, the following lemma is obvious.

Lemma 5.1. Let $z(\lambda)=\left(z_{\lambda, i}\right)_{i \in \mathbb{Z}}$ be the 01-sequence of a partition $\lambda$. Then, $\lambda$ has an inner (resp. outer) corner with content $k$ if and only if $z_{\lambda, k-1}=0, z_{\lambda, k}=1$ (resp. $\left.z_{\lambda, k-1}=1, z_{\lambda, k}=0\right)$.

For each partition $\lambda$ let $\mathcal{C}(\lambda)$ be the multiset of its contents. We explicitly compute how contents change when adding a box to one of the quotients in the Littlewood decomposition.

Lemma 5.2. Suppose that $\mu$ is a given t-core partition. Let

$$
\lambda=\left(\mu ; \lambda^{0}, \lambda^{1}, \ldots, \lambda^{i-1}, \lambda^{i}, \lambda^{i+1}, \ldots, \lambda^{t-1}\right)
$$

and

Then,

$$
\lambda^{+}=\left(\mu ; \lambda^{0}, \lambda^{1}, \ldots, \lambda^{i-1}, \lambda^{i} \cup\left\{\square_{i}\right\}, \lambda^{i+1}, \ldots, \lambda^{t-1}\right) .
$$

$$
\mathcal{C}\left(\lambda^{+}\right) \backslash \mathcal{C}(\lambda)=\left\{c_{\square_{i}} t+b_{i}, c_{\square_{i}} t+b_{i}-1, \ldots, c_{\square_{i}} t+b_{i}-(t-1)\right\} .
$$

Proof. Suppose that the 01-sequence of $\lambda$ is $\left(z_{\lambda, j}\right)_{j \in \mathbb{Z}}$. For each $0 \leq i \leq t-1$ let $\left(z_{\lambda^{i}, j}\right)_{j \in \mathbb{Z}}$ be the 01-sequence of $\lambda^{i}$. Then, $z_{\lambda^{i}, j}=z_{\lambda, j t+b_{i}}$ since $\lambda_{t \text {-core }}=\mu$. Notice that $b_{i}$ are determined by $\mu$. Let $c=c_{\square_{i}}$ be the content of $\square_{i}$. Then, by Lemma 5.1 adding the box $\square_{i}$ to $\lambda^{i}$ means that $\left(z_{\lambda^{i}, c-1}, z_{\lambda^{i}, c}\right)$, which is equal to $(0,1)$ in the 01sequence of $\lambda^{i}$, is changed to $(1,0)$; or equivalently, $\left(z_{\lambda,(c-1) t+b_{i}}, z_{\lambda, c t+b_{i}}\right)=(0,1)$ in the 01 -sequence of $\lambda$ is changed to $(1,0)$. Then by Lemma 1.1 this means that we add boxes with contents

$$
c t+b_{i}, c t+b_{i}-1, \ldots, c t+b_{i}-(t-1)
$$

to $\lambda$.

By induction and Lemma 5.2 we obtain the following lemma.

Lemma 5.3. Suppose that $\mu$ is a given t-core partition. Then

$$
\mathcal{C}(\lambda) \backslash \mathcal{C}(\mu)=\bigcup_{i=0}^{t-1}\left\{t c_{\square_{i}}+b_{i}-j: 0 \leq j \leq t-1, \square_{i} \in \lambda^{i}\right\}
$$

for every partition $\lambda$ with $\lambda_{t \text {-core }}=\mu$.

For the partition $\lambda$ and $0 \leq i \leq t-1$, let $x_{i, l}\left(0 \leq l \leq m_{i}\right)$ be the contents of inner corners of $\lambda^{i}$ and $y_{i, l}\left(1 \leq l \leq m_{i}\right)$ be the contents of outer corners of $\lambda^{i}$. 
Lemma 5.4. Suppose that $\mu$ is a given t-core partition, $r$ is a given integer, $1 \leq$ $k \leq t-1$, and $0 \leq i \leq t-1$. Let

$$
\lambda=\left(\mu ; \lambda^{0}, \lambda^{1}, \ldots, \lambda^{i-1}, \lambda^{i}, \lambda^{i+1}, \ldots, \lambda^{t-1}\right)
$$

and

$$
\lambda^{+}=\left(\mu ; \lambda^{0}, \lambda^{1}, \ldots, \lambda^{i-1}, \lambda^{i} \cup\left\{\square_{i}\right\}, \lambda^{i+1}, \ldots, \lambda^{t-1}\right) .
$$

Then

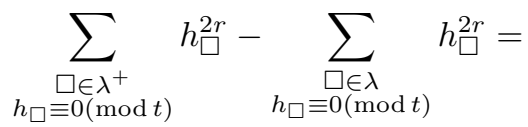

$$
\begin{aligned}
& t^{2 r}+\sum_{0 \leq l \leq m_{i}}\left(t\left(c_{\square_{i}}-x_{i, l}\right)\right)^{2 r}-\sum_{1 \leq l \leq m_{i}}\left(t\left(c_{\square_{i}}-y_{i, l}\right)\right)^{2 r}
\end{aligned}
$$

and

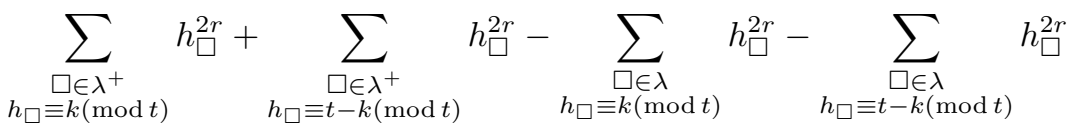

$$
\begin{aligned}
& =\sum_{0 \leq l \leq m_{i^{\prime}}}\left(t c_{\square_{i}}+b_{i}-t x_{i^{\prime}, l}-b_{i^{\prime}}\right)^{2 r}-\sum_{1 \leq l \leq m_{i^{\prime}}}\left(t c_{\square_{i}}+b_{i}-t y_{i^{\prime}, l}-b_{i^{\prime}}\right)^{2 r} \\
& +\sum_{0 \leq l \leq m_{i^{\prime \prime}}}\left(t c_{\square_{i}}+b_{i}-t x_{i^{\prime \prime}, l}-b_{i^{\prime \prime}}\right)^{2 r}-\sum_{1 \leq l \leq m_{i^{\prime \prime}}}\left(t c_{\square_{i}}+b_{i}-t y_{i^{\prime \prime}, l}-b_{i^{\prime \prime}}\right)^{2 r}
\end{aligned}
$$

where $0 \leq i^{\prime}, i^{\prime \prime} \leq t-1$ satisfy $i^{\prime} \equiv i+k(\bmod t)$ and $i^{\prime \prime} \equiv i-k(\bmod t)$. Furthermore,

$$
\begin{aligned}
\sum_{\square \in \lambda^{+}} h_{\square}^{2 r}-\sum_{\square \in \lambda} h_{\square}^{2 r} & =t^{2 r}+\sum_{j=0}^{t-1}\left(\sum_{0 \leq l \leq m_{j}}\left(t c_{\square_{i}}+b_{i}-t x_{j, l}-b_{j}\right)^{2 r}\right. \\
& \left.-\sum_{1 \leq l \leq m_{j}}\left(t c_{\square_{i}}+b_{i}-t y_{j, l}-b_{j}\right)^{2 r}\right) .
\end{aligned}
$$

Proof. Suppose that the 01-sequence of $\lambda$ is $\left(z_{\lambda, j}\right)_{j \in \mathbb{Z}}$. For each $0 \leq i \leq t-1$, let $\left(z_{\lambda^{i}, j}\right)_{j \in \mathbb{Z}}$ be the 01-sequence of $\lambda^{i}$. Then, by the Littlewood decomposition we have $z_{\lambda^{i}, j}=z_{\lambda, j t+b_{i}}$. Let $c=c_{\square_{i}}=x_{i, w_{i}}$. By Lemma 5.1, adding the box $\square_{i}$ to $\lambda^{i}$ means that $\left(z_{\lambda^{i}, c-1}, z_{\lambda^{i}, c}\right)=(0,1)$ in the 01-sequence of $\lambda^{i}$ is changed to $(1,0)$, or equivalently, $\left(z_{\lambda,(c-1) t+b_{i}}, z_{\lambda, c t+b_{i}}\right)=(0,1)$ in the 01-sequence of $\lambda$ is changed to $\left(z_{\lambda^{+},(c-1) t+b_{i}}, z_{\lambda^{+}, c t+b_{i}}\right)=(1,0)$. By Lemma 1.1, to see the difference between hook length sets of $\lambda^{+}$and $\lambda$, we just need to consider the different pairs $\left(j, j^{\prime}\right)$ where $j<j^{\prime},(1,0)=\left(z_{\lambda, j}, z_{\lambda, j^{\prime}}\right)$ or $\left(z_{\lambda^{+}, j}, z_{\lambda^{+}, j^{\prime}}\right)$, and $\left\{j, j^{\prime}\right\} \bigcap\left\{(c-1) t+b_{i}, c t+b_{i}\right\} \neq \emptyset$ in the 01-sequences of $\lambda$ and $\lambda^{+}$.

Case (i). First consider such pairs $\left(j, j^{\prime}\right)$ satisfying $j^{\prime}-j \equiv 0(\bmod t)$. Since $\left(z_{\lambda,(c-1) t+b_{i}}, z_{\lambda, c t+b_{i}}\right)=(0,1)$ and $\left(z_{\lambda^{+},(c-1) t+b_{i}}, z_{\lambda^{+}, c t+b_{i}}\right)=(1,0)$, it follows from Lemmas 1.1 and 5.1 that the hook lengths of $\lambda^{+}$for such pairs $\left(j, j^{\prime}\right)$ are

$$
\begin{gathered}
t, t\left(c-x_{i, l}\right), t\left(c-x_{i, l}-1\right), \cdots, t\left(c-y_{i, l+1}+1\right) \quad\left(0 \leq l \leq w_{i}-1\right), \\
t\left(x_{i, l}-c\right), t\left(x_{i, l}-c-1\right), \cdots, t\left(y_{i, l}-c+1\right) \quad\left(w_{i}+1 \leq l \leq m_{i}\right),
\end{gathered}
$$

and the hook lengths of $\lambda$ for such pairs are

$$
\begin{gathered}
t\left(c-x_{i, l}-1\right), t\left(c-x_{i, l}-2\right), \cdots, t\left(c-y_{i, l+1}\right) \quad\left(0 \leq l \leq w_{i}-1\right), \\
t\left(x_{i, l}-c-1\right), t\left(x_{i, l}-c-2\right), \cdots, t\left(y_{i, l}-c\right) \quad\left(w_{i}+1 \leq l \leq m_{i}\right) .
\end{gathered}
$$


Let $|a|$ be the absolute value for every real number $a$. Thus

$$
\begin{aligned}
& \left\{h_{\square} \in \mathcal{H}\left(\lambda^{+}\right): h_{\square} \equiv 0(\bmod t)\right\} \backslash\left\{h_{\square} \in \mathcal{H}(\lambda): h_{\square} \equiv 0(\bmod t)\right\} \\
& \quad=\left(\{t\} \bigcup\left\{t\left(c-x_{i, l}\right): 0 \leq l \leq w_{i}-1\right\} \bigcup\left\{t\left(x_{i, l}-c\right): w_{i}+1 \leq l \leq m_{i}\right\}\right) \\
& \backslash\left(\left\{t\left(c-y_{i, l+1}\right): 0 \leq l \leq w_{i}-1\right\} \bigcup\left\{t\left(y_{i, l}-c\right): w_{i}+1 \leq l \leq m_{i}\right\}\right) \\
& \quad=\left(\{t\} \bigcup\left\{\left|t\left(c-x_{i, l}\right)\right|: 0 \leq l \leq m_{i}, l \neq w_{i}\right\}\right) \backslash\left\{\left|t\left(c-y_{i, l+1}\right)\right|: 1 \leq l \leq m_{i}\right\},
\end{aligned}
$$

which means that

$$
\begin{aligned}
& \sum_{\substack{\square \in \lambda^{+} \\
h_{\square} \equiv 0(\bmod t)}} h_{\square}^{2 r}-\sum_{\substack{\square \in \lambda \\
h_{\square} \equiv 0(\bmod t)}} h_{\square}^{2 r}= \\
& t^{2 r}+\sum_{0 \leq l \leq m_{i}}\left(t\left(c-x_{i, l}\right)\right)^{2 r}-\sum_{1 \leq l \leq m_{i}}\left(t\left(c-y_{i, l}\right)\right)^{2 r}
\end{aligned}
$$

since $c=x_{i, w_{i}}$.

Case (ii). For $1 \leq k \leq t-1$ consider such pairs $\left(j, j^{\prime}\right)$ satisfying $j^{\prime}-j \equiv$ $k$ or $t-k(\bmod t)$. Let $0 \leq i^{\prime}, i^{\prime \prime} \leq t-1$ satisfy $i^{\prime} \equiv i+k(\bmod t)$ and $i^{\prime \prime} \equiv i-k(\bmod t)$. Then, by the similar argument as in Case (i) we obtain

$$
\begin{aligned}
& \left(\left\{h_{\square} \in \mathcal{H}\left(\lambda^{+}\right): h_{\square} \equiv k(\bmod t)\right\} \bigcup\left\{h_{\square} \in \mathcal{H}\left(\lambda^{+}\right): h_{\square} \equiv t-k(\bmod t)\right\}\right) \\
& \backslash\left(\left\{h_{\square} \in \mathcal{H}(\lambda): h_{\square} \equiv k(\bmod t)\right\} \bigcup\left\{h_{\square} \in \mathcal{H}(\lambda): h_{\square} \equiv t-k(\bmod t)\right\}\right) \\
& =\left(\left\{\left|t c-t x_{i^{\prime}, l}+b_{i}-b_{i^{\prime}}\right|: 0 \leq l \leq m_{i^{\prime}}\right\} \bigcup\left\{\left|t c-t x_{i^{\prime \prime}, l}+b_{i}-b_{i^{\prime \prime}}\right|: 0 \leq l \leq m_{i^{\prime \prime}}\right\}\right) \\
& \backslash\left(\left\{\left|t c-t y_{i^{\prime}, l}+b_{i}-b_{i^{\prime}}\right|: 1 \leq l \leq m_{i^{\prime}}\right\} \bigcup\left\{\left|t c-t y_{i^{\prime \prime}, l}+b_{i}-b_{i^{\prime \prime}}\right|: 1 \leq l \leq m_{i^{\prime \prime}}\right\}\right),
\end{aligned}
$$

which means that

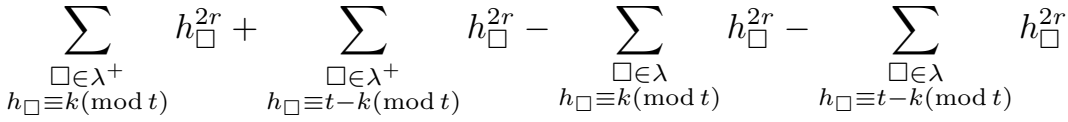

$$
\begin{aligned}
& =\sum_{0 \leq l \leq m_{i^{\prime}}}\left(t c+b_{i}-t x_{i^{\prime}, l}-b_{i^{\prime}}\right)^{2 r}-\sum_{1 \leq l \leq m_{i^{\prime}}}\left(t c+b_{i}-t y_{i^{\prime}, l}-b_{i^{\prime}}\right)^{2 r} \\
& +\sum_{0 \leq l \leq m_{i^{\prime \prime}}}\left(t c+b_{i}-t x_{i^{\prime \prime}, l}-b_{i^{\prime \prime}}\right)^{2 r}-\sum_{1 \leq l \leq m_{i^{\prime \prime}}}\left(t c+b_{i}-t y_{i^{\prime \prime}, l}-b_{i^{\prime \prime}}\right)^{2 r} .
\end{aligned}
$$

Finally, by Cases (i) and (ii) the proof is completed.

Now we are ready to prove Theorem 1.3.

Proof of Theorem 1.3. By Lemmas 5.2 and 5.4, the following two functions of partitions

and

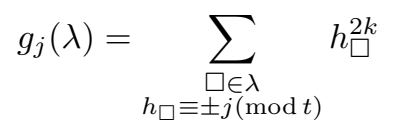

$$
g_{j}^{\prime}(\lambda)=\sum_{\substack{\square \in \lambda \\ c_{\square} \equiv j(\bmod t)}} c_{\square}^{k}
$$

are $\mu$-admissible for any $t$-core partition $\mu$, nonnegative integer $k$, and $0 \leq j \leq t-1$. Then, the proof is achieved by Theorem 3.4. 


\section{EXPlicit FORMUlas FOR THE SQUARE CASE}

Now we are able to deduce more explicit results.

Theorem 6.1. Suppose that $\mu$ is a given $t$-core partition. Recall that $b_{i}$ and $d_{i}$ are defined as in Section 4. Let $1 \leq k \leq t-1$. For every partition $\lambda$ with $\lambda_{t \text {-core }}=\mu$ we have

$$
\begin{aligned}
& \sum_{\substack{\square \in \lambda \\
h_{\square} \equiv k(\bmod t)}} h_{\square}^{2}+\sum_{\substack{\square \in \lambda \\
h_{\square} \equiv t-k(\bmod t)}} h_{\square}^{2}-\left(\sum_{\substack{\square \in \lambda \\
c_{\square} \equiv k(\bmod t)}} c_{\square}^{2}+\sum_{\substack{\square \in \lambda \\
c_{\square} \equiv t-k(\bmod t)}} c_{\square}^{2}\right) \\
&= \sum_{\substack{\square \in \mu \\
h_{\square} \equiv k(\bmod t)}} h_{\square}^{2}+\sum_{\substack{\square \in \mu \\
h_{\square} \equiv t-k(\bmod t)}} c_{\square}^{2}+\left(\sum_{\substack{\square \in \mu \\
c_{\square} \equiv k(\bmod t)}} c_{\square}^{2}\right) \\
&+\sum_{\substack{\square, j \\
c_{\square} \equiv t-k(\bmod t)}}\left(2 t^{2} n_{i} n_{j}+t\left(b_{j}+j-2 b_{i}\right) d_{j} n_{i}+t\left(b_{i}+i-2 b_{j}\right) d_{i} n_{j}\right. \\
&\left.-\frac{1}{3} t^{2}\left(d_{j} q_{3}\left(\lambda^{i}\right)+d_{i} q_{3}\left(\lambda^{j}\right)\right)\right), \\
& \text { where } n_{i}=\mid \lambda^{i} \text { for } 0 \leq i \leq t-1 .
\end{aligned}
$$

Proof. We will prove this result by induction on the size of $\lambda$. When $\lambda=\mu$, we have $\lambda^{i}=\emptyset, n_{i}=0$ for $0 \leq i \leq t-1$ and thus the identity holds. Now suppose that the identity holds for

$$
\lambda=\left(\mu ; \lambda^{0}, \lambda^{1}, \ldots, \lambda^{i-1}, \lambda^{i}, \lambda^{i+1}, \ldots, \lambda^{t-1}\right) .
$$

We just need to prove it for

$$
\lambda^{+}=\left(\mu ; \lambda^{0}, \lambda^{1}, \ldots, \lambda^{i-1}, \lambda^{i} \cup\left\{\square_{i}\right\}, \lambda^{i+1}, \ldots, \lambda^{t-1}\right) .
$$

Let $0 \leq i^{\prime}, i^{\prime \prime} \leq t-1$ satisfy $i^{\prime} \equiv i+k(\bmod t)$ and $i^{\prime \prime} \equiv i-k(\bmod t)$. Set $x_{i, w_{i}}=c_{\square_{i}}$. By Lemma 5.4, we have

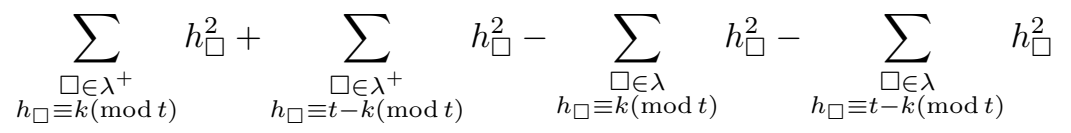

$$
\begin{aligned}
& =\sum_{0 \leq l \leq m_{i^{\prime}}}\left(t x_{i, w_{i}}+b_{i}-t x_{i^{\prime}, l}-b_{i^{\prime}}\right)^{2}-\sum_{1 \leq l \leq m_{i^{\prime}}}\left(t x_{i, w_{i}}+b_{i}-t y_{i^{\prime}, l}-b_{i^{\prime}}\right)^{2} \\
& +\sum_{0 \leq l \leq m_{i^{\prime \prime}}}\left(t x_{i, w_{i}}+b_{i}-t x_{i^{\prime \prime}, l}-b_{i^{\prime \prime}}\right)^{2}-\sum_{1 \leq l \leq m_{i^{\prime \prime}}}\left(t x_{i, w_{i}}+b_{i}-t y_{i^{\prime \prime}, l}-b_{i^{\prime \prime}}\right)^{2} \\
& =\left(t x_{i, w_{i}}+b_{i}-b_{i^{\prime}}\right)^{2}-2 t\left(t x_{i, w_{i}}+b_{i}-b_{i^{\prime}}\right)\left(\sum_{0 \leq l \leq m_{i^{\prime}}} x_{i^{\prime}, l}-\sum_{1 \leq l \leq m_{i^{\prime}}} y_{i^{\prime}, l}\right) \\
& +t^{2}\left(\sum_{0 \leq l \leq m_{i^{\prime}}} x_{i^{\prime}, l}^{2}-\sum_{1 \leq l \leq m_{i^{\prime}}} y_{i^{\prime}, l}^{2}\right) \\
& +\left(t x_{i, w_{i}}+b_{i}-b_{i^{\prime \prime}}\right)^{2}-2 t\left(t x_{i, w_{i}}+b_{i}-b_{i^{\prime \prime}}\right)\left(\sum_{0 \leq l \leq m_{i^{\prime \prime}}} x_{i^{\prime \prime}, l}-\sum_{1 \leq l \leq m_{i^{\prime \prime}}} y_{i^{\prime \prime}, l}\right) \\
& +t^{2}\left(\sum_{0 \leq l \leq m_{i^{\prime \prime}}} x_{i^{\prime \prime}, l}^{2}-\sum_{1 \leq l \leq m_{i^{\prime \prime}}} y_{i^{\prime \prime}, l}^{2}\right)
\end{aligned}
$$




$$
=\left(t x_{i, w_{i}}+b_{i}-b_{i^{\prime}}\right)^{2}+\left(t x_{i, w_{i}}+b_{i}-b_{i^{\prime \prime}}\right)^{2}+2 t^{2} n_{i^{\prime}}+2 t^{2} n_{i^{\prime \prime}} .
$$

The last equality is due to Theorem 3.1(1). On the other hand, by Lemma 5.2 we have

$$
\begin{aligned}
& \sum_{\substack{\square \in \lambda^{+} \\
c \square \equiv k(\bmod t)}} c_{\square}^{2}+\sum_{\substack{\square \in \lambda^{+} \\
c \square \equiv t-k(\bmod t)}} c_{\square}^{2}-\left(\sum_{\substack{\square \in \lambda \\
c \square \equiv k(\bmod t)}} c_{\square}^{2}+\sum_{\substack{\square \in \lambda \\
c_{\square} \equiv t-k(\bmod t)}} c_{\square}^{2}\right) \\
=\left(t x_{i, w_{i}}+b_{i}-i^{\prime}\right)^{2}+\left(t x_{i, w_{i}}+b_{i}-i^{\prime \prime}\right)^{2} . &
\end{aligned}
$$

By Theorem 3.1(3) we have

$$
q_{3}\left(\lambda^{i} \cup\left\{\square_{i}\right\}\right)-q_{3}\left(\lambda^{i}\right)=6 x_{i, w_{i}} .
$$

Finally, after putting those identities together we know the theorem is also true for $\lambda^{+}$and thus prove the claim.

We now turn to a theorem still focused on one partition at a time, but this time summing over contents or hook lengths divisible by $t$.

Theorem 6.2. Suppose that $\mu$ is a given t-core partition. For every partition $\lambda$ with $\lambda_{t \text {-core }}=\mu$ we have

$$
\begin{gathered}
\sum_{\substack{\square \in \lambda \\
h_{\square} \equiv 0(\bmod t)}} h_{\square}^{2}-\sum_{\substack{\square \in \lambda \\
c_{\square} \equiv 0(\bmod t)}} c_{\square}^{2} \\
=t^{2} \sum_{i=0}^{t-1}\left(n_{i}^{2}-d_{i}^{2} n_{i}-\frac{1}{3} d_{i} q_{3}\left(\lambda^{i}\right)\right)-\sum_{\substack{\square \in \mu \\
c_{\square} \equiv 0(\bmod t)}} c_{\square}^{2},
\end{gathered}
$$

where $n_{i}=\left|\lambda^{i}\right|$ for $0 \leq i \leq t-1$.

Proof. We will prove this result by induction on the size of $\lambda$. Notice that $\mu(0)=$ $\{h \in \mathcal{H}(\mu) \mid h \equiv 0(\bmod t)\}=\emptyset$ since $\mu$ is a $t$-core partition. When $\lambda=\mu$, we have $\lambda^{i}=\emptyset, n_{i}=0$ for $0 \leq i \leq t-1$ and thus the identity holds. Now suppose that the identity holds for

$$
\lambda=\left(\mu ; \lambda^{0}, \lambda^{1}, \ldots, \lambda^{i-1}, \lambda^{i}, \lambda^{i+1}, \ldots, \lambda^{t-1}\right) .
$$

We just need to prove it for

$$
\lambda^{+}=\left(\mu ; \lambda^{0}, \lambda^{1}, \ldots, \lambda^{i-1}, \lambda^{i} \cup\left\{\square_{i}\right\}, \lambda^{i+1}, \ldots, \lambda^{t-1}\right) .
$$

Let $x_{i, w_{i}}=c_{\square_{i}}$. By Lemma 5.4 we have

$$
\begin{aligned}
& \sum_{\substack{\square \in \lambda^{+} \\
h_{\square} \equiv 0(\bmod t)}} h_{\square}^{2}-\sum_{\substack{\square \in \lambda \\
h_{\square} \equiv 0(\bmod t)}} h_{\square}^{2} \\
= & t^{2}+\sum_{0 \leq l \leq m_{i}}\left(t x_{i, w_{i}}-t x_{i, l}\right)^{2}-\sum_{1 \leq l \leq m_{i}}\left(t x_{i, w_{i}}-t y_{i, l}\right)^{2} \\
= & t^{2}+\left(t x_{i, w_{i}}\right)^{2}-2 t^{2} x_{i, w_{i}}\left(\sum_{0 \leq l \leq m_{i}} x_{i, l}-\sum_{1 \leq l \leq m_{i}} y_{i, l}\right) \\
& +t^{2}\left(\sum_{0 \leq l \leq m_{i}} x_{i, l}^{2}-\sum_{1 \leq l \leq m_{i}} y_{i, l}^{2}\right)
\end{aligned}
$$




$$
=t^{2}+t^{2} x_{i, w_{i}}^{2}+2 t^{2} n_{i} .
$$

The last equality is due to Theorem 3.1(1). Also, by Lemma 5.2 we have

$$
\sum_{\substack{\square \in \lambda^{+} \\ c_{\square} \equiv 0(\bmod t)}} c_{\square}^{2}-\sum_{\substack{\square \in \lambda \\ c \square \equiv 0(\bmod t)}} c_{\square}^{2}=t^{2}\left(x_{i, w_{i}}+d_{i}\right)^{2} .
$$

By Theorem 3.1(3) we have

$$
q_{3}\left(\lambda^{i} \cup\left\{\square_{i}\right\}\right)-q_{3}\left(\lambda^{i}\right)=6 x_{i, w_{i}} .
$$

Finally, putting these identities together, we prove the claim.

It is clear that Theorem 1.4 is a consequence of Theorems 6.1 and 6.2 by letting $\mu=\emptyset$.

Theorem 1.6 is on $t$-Plancherel averages of square sums of congruent hook lengths.

Proof of Theorem 1.6. For two partitions

$$
\lambda=\left(\mu ; \lambda^{0}, \lambda^{1}, \ldots, \lambda^{i-1}, \lambda^{i}, \lambda^{i+1}, \ldots, \lambda^{t-1}\right)
$$

and

$$
\lambda^{+}=\left(\mu ; \lambda^{0}, \lambda^{1}, \ldots, \lambda^{i-1}, \lambda^{i} \cup\left\{\square_{i}\right\}, \lambda^{i+1}, \ldots, \lambda^{t-1}\right),
$$

by Case (ii) of Lemma 5.4 we have

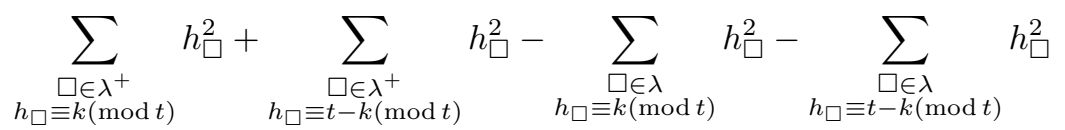

$$
\begin{aligned}
& =\sum_{0 \leq l \leq m_{i^{\prime}}}\left(t c_{\square_{i}}+b_{i}-t x_{i^{\prime}, l}-b_{i^{\prime}}\right)^{2}-\sum_{1 \leq l \leq m_{i^{\prime}}}\left(t c_{\square_{i}}+b_{i}-t y_{i^{\prime}, l}-b_{i^{\prime}}\right)^{2} \\
& +\sum_{0 \leq l \leq m_{i^{\prime \prime}}}\left(t c_{\square_{i}}+b_{i}-t x_{i^{\prime \prime}, l}-b_{i^{\prime \prime}}\right)^{2}-\sum_{1 \leq l \leq m_{i^{\prime \prime}}}\left(t c_{\square_{i}}+b_{i}-t y_{i^{\prime \prime}, l}-b_{i^{\prime \prime}}\right)^{2}
\end{aligned}
$$

where $i^{\prime} \equiv i+k(\bmod t)$ and $i^{\prime \prime} \equiv i-k(\bmod t)$.

Let

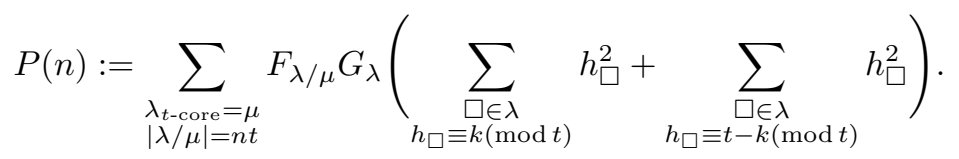

Then, by Lemma 2.2 and Theorems 3.1, 3.2 we have

$$
\begin{aligned}
& P(n+1)-P(n) \\
= & \frac{1}{t} \sum_{\substack{\lambda_{t-\text { core }=\mu} \\
|\lambda / \mu|=n t}} F_{\lambda / \mu} G_{\lambda} \sum_{i=0}^{t-1} \sum_{\lambda^{i+}=\lambda^{i} \cup\left\{\square_{i}\right\}} \frac{H_{\lambda^{i}}}{H_{\lambda^{i+}}} \\
& \left(\sum_{0 \leq l \leq m_{i^{\prime}}}\left(t c_{\square_{i}}+b_{i}-t x_{i^{\prime}, l}-b_{i^{\prime}}\right)^{2}-\sum_{1 \leq l \leq m_{i^{\prime}}}\left(t c_{\square_{i}}+b_{i}-t y_{i^{\prime}, l}-b_{i^{\prime}}\right)^{2}\right. \\
& \left.+\sum_{0 \leq l \leq m_{i^{\prime \prime}}}\left(t c_{\square_{i}}+b_{i}-t x_{i^{\prime \prime}, l}-b_{i^{\prime \prime}}\right)^{2}-\sum_{1 \leq l \leq m_{i^{\prime \prime}}}\left(t c_{\square_{i}}+b_{i}-t y_{i^{\prime \prime}, l}-b_{i^{\prime \prime}}\right)^{2}\right)
\end{aligned}
$$




$$
\begin{aligned}
= & \frac{1}{t} \sum_{\substack{\lambda_{t-\text { core }}=\mu \\
|\lambda / \mu|=n t}} F_{\lambda / \mu} G_{\lambda} \sum_{i=0}^{t-1} \sum_{\lambda^{i+}=\lambda^{i} \cup\left\{\square_{i}\right\}} \frac{H_{\lambda^{i}}}{H_{\lambda^{i+}}} \\
& \quad\left(2 t^{2} c_{\square_{i}}^{2}+2 t^{2}\left|\lambda^{i^{\prime}}\right|+2 t^{2}\left|\lambda^{i^{\prime \prime}}\right|+\left(b_{i}-b_{i^{\prime}}\right)^{2}+\left(b_{i}-b_{i^{\prime \prime}}\right)^{2}\right) \\
= & 2 t n+2 t n+2 t n+\frac{1}{t} \sum_{i=0}^{t-1}\left(\left(b_{i}-b_{i^{\prime}}\right)^{2}+\left(b_{i}-b_{i^{\prime \prime}}\right)^{2}\right) .
\end{aligned}
$$

On the other hand,

$$
\begin{aligned}
& \frac{1}{t} \sum_{i=0}^{t-1}\left(\left(b_{i}-b_{i^{\prime}}\right)^{2}+\left(b_{i}-b_{i^{\prime \prime}}\right)^{2}\right) \\
= & \frac{2}{t} \sum_{(i, j) \in B_{k}}\left(b_{i}-b_{j}\right)^{2} \\
= & \frac{2}{t} \sum_{(i, j) \in B_{k}}\left(t^{2}\left(d_{i}-d_{j}\right)^{2}+(i-j)^{2}+2 t(i-j)\left(d_{i}-d_{j}\right)\right) \\
= & 2 k(t-k)+4 t(|\mu(k)|+|\mu(t-k)|) .
\end{aligned}
$$

The last equality is due to (4.2) and Lemma 4.2. Finally,

$$
P(n+1)-P(n)=6 n t+2 k(t-k)+4 t|\mu(k)|+4 t|\mu(t-k)| .
$$

Thus,

$$
\begin{aligned}
P(n)= & 6 t\left(\begin{array}{l}
n \\
2
\end{array}\right)+(2 k(t-k)+4 t|\mu(k)|+4 t|\mu(t-k)|) n+P(0) \\
= & 6 t\left(\begin{array}{l}
n \\
2
\end{array}\right)+(2 k(t-k)+4 t|\mu(k)|+4 t|\mu(t-k)|) n \\
& +\left(\sum_{\substack{\square \in \mu \\
h_{\square} \equiv k(\bmod t)}} h_{\square}^{2}+\sum_{\substack{\square \in \mu \\
h_{\square} \equiv t-k(\bmod t)}} h_{\square}^{2}\right) .
\end{aligned}
$$

Theorem 1.7 is analogous to Theorem 1.6 when looking at $t$-Plancherel averages of square sums of hook lengths, but only the lengths divisible by $t$ are taken into account.

Proof of Theorem 1.7. For two partitions

$$
\lambda=\left(\mu ; \lambda^{0}, \lambda^{1}, \ldots, \lambda^{i-1}, \lambda^{i}, \lambda^{i+1}, \ldots, \lambda^{t-1}\right)
$$

and

$$
\lambda^{+}=\left(\mu ; \lambda^{0}, \lambda^{1}, \ldots, \lambda^{i-1}, \lambda^{i} \cup\left\{\square_{i}\right\}, \lambda^{i+1}, \ldots, \lambda^{t-1}\right),
$$

by Case (i) of Lemma 5.4 we have

Let

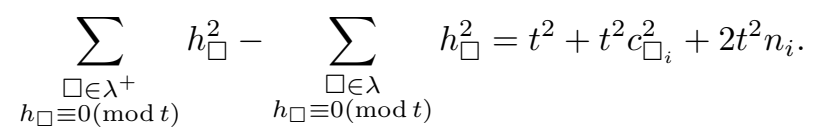

$$
P(n):=\sum_{\substack{\lambda t \text {-core }=\mu \\|\lambda / \mu|=n t}} F_{\lambda / \mu} G_{\lambda} \sum_{\substack{\square \in \lambda \\ h_{\square} \equiv 0(\bmod t)}} h_{\square}^{2} .
$$


Then,

$$
\begin{aligned}
P(n+1)-P(n) & =\frac{1}{t} \sum_{\substack{\lambda_{t-\text { core }=\mu}=\mu \\
|\lambda / \mu|=n t}} F_{\lambda / \mu} G_{\lambda} \sum_{i=0}^{t-1} \sum_{\lambda^{i+}=\lambda^{i} \cup\left\{\square_{i}\right\}} \frac{H_{\lambda}}{H_{\lambda^{i+}}}\left(t^{2}+t^{2} c_{\square_{i}}^{2}+2 t^{2} n_{i}\right) \\
& =t^{2}+3 t \sum_{i=0}^{t-1} n_{i} \\
& =t^{2}+3 n t .
\end{aligned}
$$

Thus, we have

$$
P(n)=n t^{2}+3 t\left(\begin{array}{l}
n \\
2
\end{array}\right)+P(0)=n t^{2}+3 t\left(\begin{array}{l}
n \\
2
\end{array}\right) .
$$

Finally, the next two theorems have similar concerns, looking at $t$-Plancherel averages, but this time for squares of contents. They are differentiated by the restriction in Theorem 6.3 or looking only at contents in one congruence class.

Theorem 6.3. Suppose that $\mu$ is a given $t$-core partition and $0 \leq k \leq t-1$. For each $0 \leq i \leq t-1$ let $i^{\prime}$ satisfy $0 \leq i^{\prime} \leq t-1$ and $i-i^{\prime} \equiv k(\bmod t)$. Then

$$
\sum_{\substack{\lambda_{t-c o r e}=\mu \\
|\lambda / \mu|=n t}} F_{\lambda / \mu} G_{\lambda} \sum_{\substack{\square \in \lambda \\
c \square \equiv k(\bmod t)}} c_{\square}^{2}=t\left(\begin{array}{l}
n \\
2
\end{array}\right)+\frac{1}{t} \sum_{i=0}^{t-1}\left(b_{i}-i^{\prime}\right)^{2} n+\sum_{\substack{\square \in \mu \\
c \square \equiv k(\bmod t)}} c_{\square}^{2} .
$$

Proof. For two partitions

$$
\lambda=\left(\mu ; \lambda^{0}, \lambda^{1}, \ldots, \lambda^{i-1}, \lambda^{i}, \lambda^{i+1}, \ldots, \lambda^{t-1}\right)
$$

and

$$
\lambda^{+}=\left(\mu ; \lambda^{0}, \lambda^{1}, \ldots, \lambda^{i-1}, \lambda^{i} \cup\left\{\square_{i}\right\}, \lambda^{i+1}, \ldots, \lambda^{t-1}\right),
$$

by Lemma 5.2 we have

$$
\sum_{\substack{\square \in \lambda^{+} \\ c \square \equiv k(\bmod t)}} c_{\square}^{2}-\sum_{\substack{\square \in \lambda \\ c \square \equiv k(\bmod t)}} c_{\square}^{2}=\left(t c_{\square}+b_{i}-i^{\prime}\right)^{2} .
$$

Let

$$
P(n):=\sum_{\substack{\lambda_{t-\text { core }}=\mu \\|\lambda / \mu|=n t}} F_{\lambda / \mu} G_{\lambda} \sum_{\substack{\square \in \lambda \\ c \square \equiv k(\bmod t)}} c_{\square}^{2} .
$$

Then, we have

$$
\begin{aligned}
P(n+1)-P(n) & =\frac{1}{t} \sum_{\substack{\lambda_{t-\text { core }}=\mu \\
|\lambda / \mu|=n t}} F_{\lambda / \mu} G_{\lambda} \sum_{i=0}^{t-1} \sum_{\lambda^{i+}=\lambda^{i} \cup\left\{\square_{i}\right\}} \frac{H_{\lambda}}{H_{\lambda^{i+}}}\left(t c_{\square_{i}}+b_{i}-i^{\prime}\right)^{2} \\
& =t n+\frac{1}{t} \sum_{i=0}^{t-1}\left(b_{i}-i^{\prime}\right)^{2}
\end{aligned}
$$

Finally, we obtain

$$
P(n)=t\left(\begin{array}{l}
n \\
2
\end{array}\right)+\frac{1}{t} \sum_{i=0}^{t-1}\left(b_{i}-i^{\prime}\right)^{2} n+P(0)
$$




$$
=t\left(\begin{array}{l}
n \\
2
\end{array}\right)+\frac{1}{t} \sum_{i=0}^{t-1}\left(b_{i}-i^{\prime}\right)^{2} n+\sum_{\substack{\square \in \mu \\
c \square \equiv k(\bmod t)}} c_{\square}^{2} .
$$

Theorem 6.4. Suppose that $\mu$ is a given $t$-core partition. Then we have

$$
\sum_{\substack{\lambda_{t-c o r e}=\mu \\
|\lambda / \mu|=n t}} F_{\lambda / \mu} G_{\lambda} \sum_{\square \in \lambda} c_{\square}^{2}=t^{2}\left(\begin{array}{l}
n \\
2
\end{array}\right)+\frac{\left(t^{3}-t\right) n}{6}+2 t n|\mu|+\sum_{\square \in \mu} c_{\square}^{2} .
$$

Proof. By Theorem 6.3 we have

$$
\begin{aligned}
& \sum_{\substack{\lambda_{t-\text { core }=\mu} \\
|\lambda / \mu|=n t}} F_{\lambda / \mu} G_{\lambda} \sum_{\square \in \lambda} c_{\square}^{2} \\
= & t^{2}\left(\begin{array}{l}
n \\
2
\end{array}\right)+\frac{1}{t} \sum_{i=0}^{t-1} \sum_{j=0}^{t-1}\left(b_{i}-j\right)^{2} n+\sum_{\square \in \mu} c_{\square}^{2} \\
= & t^{2}\left(\begin{array}{l}
n \\
2
\end{array}\right)+\frac{\left(t^{3}-t\right) n}{6}+2 t n|\mu|+\sum_{\square \in \mu} c_{\square}^{2} .
\end{aligned}
$$

The last equality is due to (4.3), (4.4) and (4.5).

\section{Acknowledgments}

P.-O.D. and H.X. acknowledge support from grant PP00P2_138906 of the Swiss National Science Foundation. H.X. also acknowledges support of a Forschungskredit FK-14-093 from the University of Zürich.

\section{REFERENCES}

[1] T. Amdeberhan, Differential operators, shifted parts, and hook lengths, Ramanujan J. 24(3) (2011), 259-271.

[2] D. Armstrong, C. Hanusa and B. Jones, Results and conjectures on simultaneous core partitions, European J. Combin. 41 (2014), 205-220.

[3] J. Bandlow, An elementary proof of the hook formula, Electron. J. Combin. 15 (2008), research paper 45.

[4] K. Carde, J. Loubert, A. Potechin, and A. Sanborn, Proof of Han's Hook Expansion Conjecture, preprint; arXiv:0808.0928.

[5] J. S. Frame, G. de B. Robinson, and R. M. Thrall, The hook graphs of $S_{n}$, Canad. J. Math. 6 (1954), 316-324.

[6] C. Greene, A. Nijenhuis and H. S. Wilf, A probabilistic proof of a formula for the number of Young tableaux of a given shape, Adv. in Math. 31 (1979), no. 1, 104-109.

[7] C. Greene, A. Nijenhuis and H. S. Wilf, Another probabilistic method in the theory of Young tableaux, J. Combin. Theory Ser. A 37 (1984), no. 2, 127-135.

[8] G.-N. Han, The Nekrasov-Okounkov hook length formula: refinement, elementary proof, extension, and applications, Ann. Inst. Fourier 60 (2010), no. 1, 1-29.

[9] G.-N. Han, Some conjectures and open problems on partition hook lengths, Experimental Mathematics 18 (2009) 97-106.

[10] G.-N. Han, Hook lengths and shifted parts of partitions, Ramanujan J. 23(1-3) (2010), $127-135$.

[11] G.-N. Han and H. Xiong, Difference operators for partitions and some applications, preprint; arXiv: 1508.00772.

[12] G. James and A. Kerber, The Representation Theory of the Symmetric Group, Encyclopedia of Mathematics and its Applications, 16. Addison-Wesley Publishing Co., Reading, Mass., 1981. 
[13] D. Knuth, The Art of Computer Programming, Vol. 3: Sorting and Searching, AddisonWesley, London, 1973, pp. 54-58.

[14] I. G. Macdonald, Symmetric functions and Hall polynomials, Oxford Mathematical Monographs, The Clarendon Press, Oxford University Press, New York, second ed., 1995.

[15] N. A. Nekrasov and A. Okounkov, Seiberg-Witten theory and random partitions, in The unity of mathematics, Progress in Mathematics 244, Birkhäuser Boston, 2006, pp. 525-596.

[16] G. Olshanski, Anisotropic Young diagrams and infinite-dimensional diffusion processes with the Jack parameter, Int. Math. Res. Not. IMRN 6 (2010), 1102-1166.

[17] G. Olshanski, Plancherel averages: Remarks on a paper by Stanley, Electron. J. Combin. 17 (2010), research paper 43.

[18] G. Panova, Polynomiality of some hook-length statistics, Ramanujan J. 27 (2012), no. 3, 349-356.

[19] G.-C. Rota, On the foundations of combinatorial theory: I. Theory of Möbius functions, $Z$. Wahrscheinlichkeitstheorie und Verw. Gebiete 2 (1964), 349-356.

[20] R. P. Stanley, Some combinatorial properties of hook lengths, contents, and parts of partitions, Ramanujan J. 23(1-3) (2010), 91-105.

[21] R. P. Stanley, Differential posets, J. Amer. Math. Soc. 1 (1988), no. 4, 919-961.

[22] R. P. Stanley, Enumerative Combinatorics, vol. 2, Cambridge University Press, New York/Cambridge, 1999.

I-Math, Universität Zürich, Winterthurerstrasse 190, Zürich 8057, Switzerland

E-mail address: paul-olivier.dehaye@math.uzh.ch

I.R.M.A., UMR 7501, Université de Strasbourg et CNRS, 7 rue René Descartes, F67084 Strasbourg, France

E-mail address: guoniu.han@unistra.fr

I-Math, Universität Zürich, Winterthurerstrasse 190, Zürich 8057, Switzerland

E-mail address: huan.xiong@math.uzh.ch 